\title{
Essai de caractérisation phytoécologique des for- mations herbacées pâturées de Grande-Terre (Guadeloupe)
}

\author{
Jacques FOURNET \& Pascal MONESTIEZ (*) \\ I.N.R.A., Station de Pathologie végétale, de Phytoécologie et de Malherbologie, Centre de Recherches des \\ Antilles et de la Guyane, 97170 Petit-Bourg (Guadeloupe)
}

(*) I.N.R.A., Laboratoire de biométrie, Centre de Recherches d'Avignon, 84140 Montfavet

RÉSUMÉ

\begin{abstract}
Dans le but de caractériser les types de prairies naturelles (ou " savanes ») de la Grande-Terre (Guadeloupe), 350 relevés ont été effectués, selon un plan d'échantillonnage stratifié en fonction de la pluviométrie et de la profondeur du sol. Outre les espèces présentes (et leurs abondances), 61 variables ont été notées pour chaque relevé. La technique d'interprétation utilisée est celle des profils écologiques, conduisant à la constitution de groupes écologiques plurifactoriels d'espèces. Il est montré que les variables du milieu les plus explicatives ressortissent au macroclimat (pluviométrie surtout) et aux caractères édaphiques et topographiques. Les communautés végétales mises en évidence sont caractérisées par leur composition en groupes écologiques, et par leurs conditions de milieu. Une présentation synthétique des résultats est faite grâce à l'Analyse Factorielle des Correspondances (AFC). Les types de savanes ainsi caractérisés sont en outre évalués par le calcul des moyennes des valeurs pastorales des relevés constitutifs. Les « paysages prairiaux » des petites régions de Grande-Terre sont décrits.
\end{abstract}

Mots clés additionnels : Prairie naturelle, milieu tropical, Caraïbes, phytoécologie, groupe écologique, valeur pastorale.

\begin{abstract}
Phytoecological characterization of grazed grasslands in Grande-Terre (Guadeloupe).
In order to characterize the types of natural meadows (or "savannas") in Grande-Terre (Guadeloupe) fig. 1-, 350 samples were taken. Sampling was stratified according to annual rainfall and soil depth. Besides the species present (and their abundance), 61 factors were noted for each sample. The results were interpreted by the ecological series method, which gives plurifactorial ecological species groups (tables la and $1 \mathrm{~b}$ ). The most explanatory environmental factors were macroclimate (mainly rainfall), and edaphic and topographic characteristics. The plant communities defined (tables $3 \mathrm{a}$ and $3 \mathrm{~b}$ ) were characterized by their composition in ecological species groups (table 2), and by their prevalent environmental conditions. These entities could be represented synthetically by Factor Analysis of Correspondences (AFC) (fig. 2 through 5). The pasture types resulting from this study were evaluated by computing the average pasture value of the component samples, according to rough quality indices of species. A "pastoral landscape" of the regions of Grande-Terre was accordingly described (table 4).
\end{abstract}

Additional key words : Natural pasture, tropics, Caribbean, plant ecology, ecological group, pasture value.

\section{INTRODUCTION}

Ce n'est que depuis peu de temps que les agronomes et zootechniciens s'intéressent au matériel végétal et animal indigène des Petites Antilles.

Pour la prairie, cet intérêt est né de la constatation qu'après des années d'une politique de plantation subventionnée d'espèces exotiques, les ressources fourragères sont encore largement constituées par les « savanes ", terme impropre mais traditionnel que nous conserverons par commodité dans ce qui suit pour dési- gner les prairies subclimaciques (« naturelles»). Le déclin de la Canne à sucre n'a fait que confirmer cet état de fait, en laissant la place à de vastes étendues enherbées.

Il est donc apparu nécessaire de tenter de mettre au point des techniques rationnelles de gestion de ces savanes : rythme d'exploitation, charge en animaux, entretien, enrichissement en légumineuses, fumure... Mais la valeur des essais menés à cet effet, c'est-à-dire la possibilité de généraliser leurs résultats, suppose la connaissance de l'objet étudié. 
Les prairies subclimaciques des Antilles ont été relativement peu étudiées. Le travail le plus élaboré est celui de GARCIA-MOLINARI (1952), sur les savanes de Porto-Rico.

Les Petites Antilles ont fait l'objet de quelques études de type écologique dans le passé ; mais ces études ont surtout porté tantôt sur la phytogéographie (STEHLÉ, 1935 ; VÉLEZ, 1957) ou la phytosociologie générale (STEHLÉ, 1937a et $b$ ), aboutissant - par leur nature même - à des résultats peu directement utilisables par l'agronome, tantôt sur une typification dans une zone très limitée (DOREAU \& VIVIER, 1979a et $b$ ), aux conclusions non généralisables. STEHLÉ (1937a, 1950, 1956) a abordé l'étude des formations herbacées de Guadeloupe avec l'œil de l'agronome; mais ses résultats sont plus des «inventaires-catalogues » d'espèces qu'un inventaire raisonné des types de prairies prenant en compte à la fois la composition floristique et les conditions de milieu. Cet auteur recommande pourtant, de façon très clairvoyante, ce dernier type d'études: «L'un des premiers objectifs scientifiques, en vue d'amélioration technique, doit être (...) l'élaboration de catégories distinctes de pâtures (...) basées sur des facteurs édapho-climatiques et des compositions (...) floristiques... » (STEHLÉ, 1951).

C'est pourquoi nous avons jugé intéressant d'entreprendre, en utilisant des techniques modernes, cette caractérisation phytoécologique, dont les premiers résultats, concernant uniquement la Grande-Terre (principale région d'élevage de la Guadeloupe), sont rapportés ici.

\section{MÉTHODE DE TRAVAIL}

\section{A. Les formations étudiées}

Le but de ce travail est de caractériser des formations herbacées pâturées (ou pâturables) ayant atteint leur point d'équilibre ; les prairies visiblement récentes ont donc été évitées. Nos observations nous conduisent à penser qu'une prairie de plus de 2 ans a pratiquement acquis ses caractères définitifs. A l'analyse des résultats, il s'est néanmoins avéré que quelques prairies « jeunes » avaient été inventoriées : elles se révèlent par leur composition floristique de type postcultural, ou dominent les " mauvaises herbes ". Ces relevés, très peu nombreux, n'entachent pas, selon nous, les conclusions de l'étude.

\section{B. Le domaine d'étude}

La Grande-Terre $\left(586 \mathrm{~km}^{2}\right)$, partie calcaire de la Guadeloupe, a maintes fois été décrite quant à sa géographie, sa géologie et son climat, en particulier par LASSERRE (1961). La figure 1 indique la division en petites régions, bien caractérisées par cet auteur, que nous avons utilisée dans la présente étude. Nous ne rappellerons ici, très sommairement, que les traits essentiels de ces petites régions, en signalant les types de sols rencontrés (nomenclature de DE CRECY, 1969 \& FAOUNESCO pour les équivalents anglais de la fig. 2).

\section{Figure 1}

Division de la Grande-Terre en petites régions. Isohyètes moyennes (1929-1978).

Division of Grande-Terre into subregions. Mean isohyets (1929. 1978).

1. Plateau Nord et Hauteurs Caraibes.

2. Plateau moyen (ou de Sainte-Marguerite).

3. Plateau Est (ou de Saint-François).

4. Grands-Fonds.

5. Collines de Gosier.

6. Plaine de Grippon (ou de Morne à l'Eau).

7. Plaine des Abymes.

8. Mangrove et arrière-mangrove.

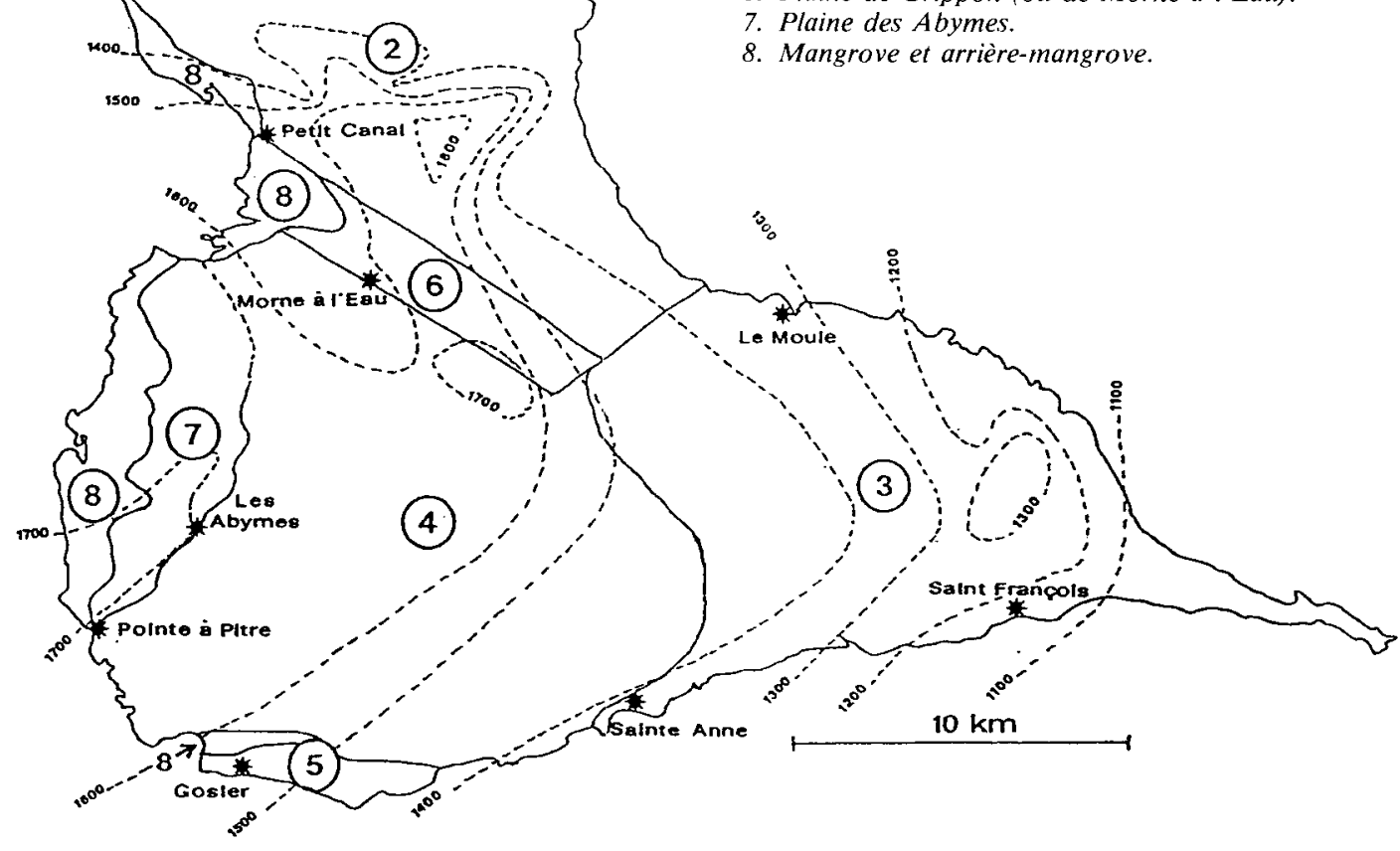




\section{Les plateaux de l'est et du nord}

(Régions 1, 2 et 3) : zones assez plates, sur calcaires blancs récifaux du miocène. Pluviométrie : de 950 à 1450 (localement 1800 ) $\mathrm{mm}$. Vertisols souvent hydromorphes dans les fonds et les parties plates, lithosols calcaires et rendzines sur les sommets, et sols intermédiaires sur les pentes : sols vertiques en bas, sols calciques mélanisés en haut de versant; sur les falaises littorales sèches, on trouve des sols fersiallitiques.

\section{Les Grands Fonds}

(Région 4) : région au relief karstique complexe, formée de surfaces d'aplanissement qui relient les sommets, coupées de nombreuses vallées sèches aux versants raides. Les calcaires sont plus anciens que ceux des plateaux : calcaires ocres à algues et éléments volcaniques, calcaires ocres subrécifaux, mis à jour par l'érosion d'un anticlinal. Pluviométrie : 1350 à $1750 \mathrm{~mm}$. Sols : Rendzines sur calcaire ou sur tuf sur les sommets et en haut des versants; sols calciques mélanisés sur les pentes; sols vertiques en bas de pente; vertisols modaux (ou plus ou moins hydromorphes) sur les calcaires ocres et les argiles gonflantes des fonds ; « vertisols complexes à apports volcaniques récents » sur les affleurements du socle de base, au raccordement avec les plaines. C'est une région de polyculture vivrière traditionnelle, mais l'élevage tend à s'y développer.

\section{La plaine de Grippon}

(Région 6) : région très plate, étroite et allongée, qui correspond vraisemblablement à un fossé tectonique au pied de l'anticlinal des Grands Fonds. Pluviométrie 1450 à $1700 \mathrm{~mm}$. D'est en ouest, on trouve successivement : des vertisols plus ou moins hydromorphes, des « vertisols complexes à apports volcaniques récents ", caractérisés par des bigarrures rouges et blanches en profondeur, et des sols hydromorphes, au passage très progressif à l'« arrière-mangrove ». Dans l'ensemble, les sols sont mal drainés. Région encore récemment cannière, convertie à l'élevage.

\section{La plaine des Abymes}

(Région 7): plaine établie sur le « complexe de base " volcanique, caractérisée par l'abondance des sols ferrallitiques. Les buttes-témoins des GrandsFonds sont fréquentes. Vers l'ouest, le passage à l' " arrière-mangrove » est progressif. Pluviométrie : 1600 à $1800 \mathrm{~mm}$. L'élevage se développe également, mais est concurrencé par l'urbanisation.

\section{Les collines de Gosier}

(Région 5) : cette région, en voie de « suburbanisation " rapide, a été étudiée surtout dans un souci d'exhaustivité. Pluviométrie : $1400-1600 \mathrm{~mm}$. Sols : comme sur les plateaux.

\section{L'arrière-mangrove}

(Région 8) : Nous désignons ainsi toutes les zones sur sols hydromorphes, en général constitués d'alluvions ou de colluvions récentes, argileuses et/ou organiques. Le plus souvent proches du littoral, ces zones subissent parfois l'influence de l'eau salée. Eclatée en un grand nombre d'unités presque tout le long du littoral, cette « région » est surtout importante en arrière de la Forêt rivulaire à Pterocarpus qui borde la mangrove du Grand et du Petit Cul-de-Sac Marin et de la Rivière Salée. Bien que présentant des conditions difficiles, c'est depuis longtemps une importante zone d'élevage traditionnel.

\section{Méthode des profils écologiques}

Pour la constitution des groupes écologiques, nous avons utilisé la méthode des profils écologiques (GoUNOT, 1969 ; GODRON, 1971; GUILLERM, 1971 ; DAGET \& GODRON, 1982), qui permet la caractérisation de chaque espèce végétale par son comportement vis-à-vis de chaque variable du milieu.

L'échantillonnage est stratifié en fonction de deux variables considérées a priori comme primordiales : la pluviométrie et la profondeur du sol ; en outre, le nombre de relevés effectués dans chaque petite région est approximativement proportionnel à sa surface.

Il a été effectué 350 relevés, chacun d'environ $100 \mathrm{~m}^{2}$ (surface considérée comme largement supérieure à l'aire minimale), dans une station apparemment homogène quant aux conditions de milieu et à l'aspect de la végétation. Les risques d'hétérogénéité sont faibles: GRUET (1984) a montré que l'homogénéité floristique des savanes à Dichanthium de Grande-Terre est beaucoup plus forte que leur homogénéité «visuelle ".

La nomenclature botanique utilisée est, à quelques mises à jour et compléments près (PROCTOR in HOWARD, 1977 ; HOWARD, 1979), celle que l'un de nous a adoptée dans la Flore de Guadeloupe et de Martinique (FOURNET, 1978).

Le recouvrement absolu de chaque espèce est évalué visuellement; il est utilisé pour le calcul de la note d'abondance (voir Analyse Factorielle des Correspondances) et de la Valeur Pastorale (voir plus loin). Les profils écologiques ne prennent pas en compte l'abondance, mais seulement la présence/absence. La technique de constitution des groupes écologiques (plurifactoriels) provisoires a déjà été décrite (FoURNET, 1983).

Les groupes -écologiques définitifs résultent de la comparaison des groupes provisoires avec les groupes cénologiques (constitués par examen des corrélations de point entre espèces), et des corrections apportées après étude des cartes factorielles de l'Analyse Factorielle des Correspondances (AFC).

Les groupements de relevés (constituant ce que nous désignerons par la suite sous le nom de « communautés végétales » ou parfois de «groupements ») sont constitués après comparaison des affinités sociologiques (corrélation de point) et écologiques (corrélation de PEARSON calculée sur les états des variables) des relevés pris deux à deux, et après examen des cartes factorielles de l'AFC.

\section{Les variables}

Soixante et une variables ont été notées dans chaque relevé. Les principales d'entre elles concernent :

- La situation générale : région, altitude, reliefsecteur. 
- Le macroclimat : précipitations annuelles, nombre de mois à pluviométrie inférieure à $50 \mathrm{~mm}$ et à $100 \mathrm{~mm}$, précipitations du mois le plus arrosé, indice d'aridité de DE MARTONNE...

- Le microclimat : orientation nord/sud et est/ouest, exposition au vent, intensité et fréquence de l'ombrage...

- La topographie : relief-station, pente moyenne, situation topographique, microrelief...

- Les relations sol-eau : fréquence de submersion, drainage externe, drainage interne...

- La physique du sol : compacité, texture, profondeur du sol meuble, roche-mère (ou substrat)...

- La chimie du sol : $\mathrm{pH}$ eau et $\mathrm{pH} \mathrm{KCl}$, teneurs en azote total et carbone total, rapport $\mathrm{C} / \mathrm{N}$, conductivité d'une suspension de pâte molle du sol...

- La structure et l'organisation de la végétation : recouvrement des différentes strates, des plantes herbacées, des graminées, des ligneux bas... Ces variables ne sont utilisées que dans la description physionomique des communautés.

- Les facteurs d'exploitation : piétinement, intensité apparente d'exploitation...

- Enfin quelques facteurs synthétiques, comme l'humidité apparente, le type de sol et le climax forestier probable.

Les liaisons entre variables ont été étudiées par examen des corrélations de Pearson entre elles; cette méthode, très critiquable, permet d'avoir rapidement une idée grossière du degré de liaison entre deux variables ; elle ne risque pas de montrer de fausses liaisons, mais au contraire de passer à côté des liaisons non linéaires.

L'efficacité d'une variable (moyenne des informations mutuelles (IM) entre la variable et les $n$ premiers taxons, classés selon les IM décroissantes - GODRON, 1971 ) est calculée pour $n=150$ (sur 324 taxons observés).

\section{E. Analyse factorielle des correspondances (AFC)}

Technique d'ordination, et non de classification, l'AFC a été, dans cette étude, utilisée essentiellement à 2 fins :

- Contrôle de la validité et de la cohérence des groupes écologiques et communautés, construits par la méthode décrite plus haut.

- Présentation très synthétique des résultats.

Plusieurs analyses ont été effectuées ; celles qui ont donné les résultats les plus facilement utilisables sont :

a) Analyse 350 relevés $\times$ abondance (notée de 1 à 6) des espèces.

b) Analyse 260 relevés "moyens » $\times$ importance dans chaque relevé des 55 groupes écologiques les plus importants.

Par relevés « moyens » nous désignons les relevés ne correspondant pas à des conditions extrêmes de sécheresse ou d'humidité.

Les groupes écologiques les plus importants sont ceux dont les espèces constitutives présentent à la fois des fréquences non négligeables et des profils écologi- ques suffisamment caractéristiques, au moins pour certaines des variables prépondérantes (définies plus loin).

L'importance de chaque groupe écologique dans chaque relevé est calculée par la formule suivante :

$$
\text { Ir }=\frac{\mathrm{Ar}}{\mathrm{A}_{\max }} \times 10
$$

où :

- Ar est la somme des abondances (notées de 0 à 6) des espèces du groupe présentes dans le relevé.

- Amax est la somme des abondances maximales de toutes les espèces du groupe dans l'ensemble des relevés.

- Ir est l'importance du groupe dans le relevé, et varie entre 0 et 10 .

On néglige ainsi le risque de biais provoqué par l'éventuelle très forte abondance d'une espèce dans un ou quelques relevés ; il aurait sans doute mieux valu utiliser la somme des abondances moyennes. Il ne nous paraît pas cependant, après réexamen des données, que ce biais ait été très important ici.

\section{F. Valeur pastorale}

La valeur pastorale (VP) d'un groupement est évaluée comme moyenne arithmétique des VP des relevés qui la composent. La VP d'un relevé est calculée selon une technique classique, rappelée par DAGET \& POISSONNET (1971) ; les contributions spécifiques sont calculées à partir des recouvrements des taxons. Les indices de qualité retenus, notés de 0 à 5 , sont le plus souvent appréciés grossièrement en fonction des connaissances des agronomes, des zootechniciens, et des éleveurs : rares sont en effet les données bibliographiques sur les espèces tropicales spontanées (XANDÉ, 1985). Certaines espèces nuisibles - en particulier épineuses ou vénéneuses (STEHLÉ, 1956 ; OAKES \& BUTCHER, 1962) - reçoivent un indice négatif.

\section{RÉSULTATS}

\section{A. Observations floristiques}

\section{Fréquences absolues des espèces}

Les taxons (espèces, sous-espèces ou variétés) présents au moins une fois dans les 350 relevés sont au nombre de $324 ; 138(42,6 \mathrm{p} .100)$ sont présents dans moins de 5 relevés, $63(19,4$ p. 100) dans 5 à 10 relevés, $88(27,2$ p. 100$)$ dans 11 à 50 relevés, $23(7$ p. 100) dans 51 à 100 relevés, et $12(3,7$ p. 100$)$ dans plus de 100 relevés.

\section{Les familles}

Les mousses terrestres n'ont pu être identifiées, et sont regroupées dans un seul «taxon » dans cette étude. Les 323 taxons de Phanérogames et Fougères appartiennent à 190 genres et 56 familles. 13 familles 
regroupent 236 (soit 73 p. 100) des taxons et 126 (soit 66 p. 100) des genres. Ce sont les suivantes, dans l'ordre d'importance décroissante (les nombres de taxons et de genres, sont indiqués entre parenthèses) :

Poaceae $(60,29)$

Fabaceae $(36,18)$

Asteraceae $(24,19)$

Cyperaceae $(22,10)$

Euphorbiaceae $(22,8)$

Convolvulaceae $(14,5)$

Malvaceae $(12,6)$

\section{Les espèces principales}

Nous désignons ainsi les taxons atteignant fréquemment de forts recouvrements absolus, et dont la proportion dans la biomasse est en général très importante :

- 113 taxons (dont 39 , soit 34 p. 100 , de Poaceae), de 75 genres et 23 familles peuvent présenter un recouvrement supérieur à 10 p. 100 ;

- 84 taxons (dont 33, soit 39 p. 100, de Poaceae), de 55 genres et 20 familles, peuvent dépasser 20 p. 100 de recouvrement ;

- 44 taxons (dont 25, soit 57 p. 100, de Poaceae), de 34 genres et 13 familles, dépassent plus ou moins fréquemment 50 p. 100 de recouvrement.

Parmi ces derniers, les taxons atteignant le plus fréquemment de très forts recouvrements sont les suivants (on notera la prépondérance des Poaceae) :

a) Dans la grande majorité des savanes « moyennes » (ni subaquatiques ni subarides)

Axonopus compressus

Dichanthium annulatum

Dichanthium aristatum

Paspalum conjugatum

Stenotaphrum secundatum

Wedelia trilobata

b) Dans les savanes très humides à subaquatiques

Brachiaria mutica

Echinochloa guadeloupensis

Eriochloa polystachya

Hymenachne amplexicaulis

Ipomoea aquatica (rare)

Panicum laxum

Paspalum distichum

Polygonum punctatum

Bacopa monnieri

Eleocharis mutata

c) Dans les savanes très sèches à subarides

Sida ciliaris

Bothriochloa pertusa

Dactyloctenium aegyptium

Sporobolus virginicus (littoral)

Stylosanthes hamata

d) Sur sol salé

Fimbristylis cymosa

Paspalum vaginatum

\section{Les légumineuses}

Si les taxons appartenant aux Fabaceae, aux Caesalpiniaceae et aux Mimosaceae sont relativement nom- breux (52), rares sont ceux qui présentent à la fois une fréquence élevée, un recouvrement notable et une valeur fourragère intéressante. Les espèces de fréquence absolue supérieure à 4 et pouvant présenter un recouvrement supérieur à 10 p. 100 sont les suivantes :

Cassia (Senna) obtusifolia

Aeschynomene americana

Aeschynomene sensitiva

Alysicarpus vaginalis

Centrosema pubescens

Crotalaria retusa

Desmodium adscendans

Desmodium axillare

Desmodium incanum (var. a)

Desmodium incanum (var. b)

Desmodium triflorum

Indigofera spicata

Indigofera tinctoria

Mimosa pudica

Neptunia plena

Phaseolus adenanthus

Stylosanthes hamata

Teramnus labialis

Vigna luteola

Parmi ces espèces, certaines ne sont pratiquement pas consommées, pour diverses raisons : odeur repoussante (Senna obtusifolia), toxicité (Crotalaria retusa), présence d'épines (Mimosa pudica), port plaqué contre le sol (Alysicarpus vaginalis, Desmodium triflorum, Indigofera spicata). Seuls le Teramnus labialis, le Vigna luteola et le Stylosanthes hamata semblent présenter un réel intérêt fourrager.

\section{Remarques concernant certaines espèces}

Le Dichanthium (ou Dichantium) caricosum (L.) Stapf a parfois été envisagé comme une "grande espèce " aux nombreuses formes (ROBERTY, 1960, suivi par FOURNET, 1978); on distingue maintenant plusieurs espèces. Il existe ainsi en Grande-Terre 2 espèces de "Petit Foin 》: Dichanthium aristatum (Poir.) C. E. Hubb. et D. annulatum (Forssk.) Stapf, faciles à distinguer (cf. DE WET \& HARLAN, 1968, GOULD in HOWARD, 1979). Le D. caricosum au sens strict ne semble pas exister ici. Dichanthium aristatum préfère les régions à pluviométrie inférieure à $1300 \mathrm{~mm}$, alors que $D$. annulatum se rencontre sous des pluviométries allant de 1250 à $1800 \mathrm{~mm}$. Cependant, s'agissant d'espèces asiatiques introduites assez récemment aux Antilles, on ne peut exclure qu'elles soient encore en période de colonisation, et que la répartition constatée ne corresponde pas à un équilibre.

Nous avons observé une espèce de Paspalidium morphologiquement et écologiquement très distincte du $P$. geminatum (Forssk.) Stapf, bien connu. Il pourrait s'agir de $P$. obtusifolium (Delile) Simpson (cf. CHIPPINDALL in MEREDITH, 1955, p. 366, illustr. p. 367) ; sans doute d'introduction assez récente, cette espèce semble actuellement en expansion en Grande-Terre. Elle n'est signalée, à notre connaissance, dans aucune autre île des Antilles.

Nous avons regretté le manque de connaissance de la communauté botanique internationale sur les Digitaria de notre zone, en particulier sur ceux du groupe ciliaris-bicornis-sanguinalis, pour lequel la clef de 
GOULD (in HOWARD, 1979) ne permet pas une discrimination efficace en pratique, pas plus que celle de HENRARD (1950).

Plusieurs espèces semblent présenter des écotypes, difficiles ou impossibles à distinguer, qui se signalent par des profils nettement bi- ou polymodaux pour certaines variables. C'est le cas par exemple de Cynodon dactylon, de Lippia nodiflora et de Desmodium incanum.

\section{B. Les variables}

\section{Liaisons entre variables}

L'étude des liaisons entre variables montre que beaucoup d'entre elles peuvent être rassemblées en grands groupes ou "facteurs " (au sens de l'analyse factorielle), dont les principaux sont :

- le macroclimat,

- un groupe synthétisant les variables édaphiques physiques et topographiques en relation avec l'eau dans le sol,

- un groupe résumant certaines propriétés chimiques du sol : $\mathrm{pH}$, réaction $\mathrm{HCl}$, texture de l'horizon superficiel,

- un groupe « Matière organique » réunissant Carbone et Azote totaux,

- un groupe «Densité du peuplement végétal » regroupant les variables notant le recouvrement (des herbacées, des Graminées...) et celles exprimant l'occupation de la surface du sol,

- un groupe "Structure de la végétation": Recouvrement des diverses strates, et Hauteur du tapis herbacé,

- les variables notant l'architecture de l'espèce dominante et des graminées ne sont liées à aucun groupe. L'exposition au vent et l'intensité d'ombrage sont liées entre elles, comme on pouvait s'y attendre, mais sont indépendantes de tous les groupes. Enfin quelques variables a priori importantes sont liées à la plupart des autres groupes, ce qui confirme leur caractère synthétique, d'ailleurs évident : c'est le cas de la Région et du Climax forestier probable.

\section{Les variables prépondérantes}

Les variables du milieu les mieux échantillonnées et dont l'« efficacité " (donc la valeur explicative à l'égard de la répartition des espèces) est la plus élevée, que nous appellerons «variables prépondérantes », sont, dans l'ordre d'efficacité décroissante :

- le climax forestier probable,

- le relief du secteur,

- la région,

- la roche-mère,

- l'humidité apparente,

- le nombre de mois à pluviométrie moyenne supérieure à $150 \mathrm{~mm}$,

- le nombre de mois à pluviométrie moyenne inférieure à $100 \mathrm{~mm}$,

- les précipitations moyennes du mois le plus arrosé,

- les précipitations annuelles moyennes,

- la fréquence de submersion,

- le type de sol,
- le nombre de mois à pluviométrie moyenne inférieure à $50 \mathrm{~mm}$,

- le drainage interne,

- la compacité de l'horizon 2 (sous l'horizon superficiel),

- la situation topographique,

- la profondeur du sol.

Elles appartiennent toutes, à l'exception de la Région et du Climax probable, qui ont une signification très synthétique, au groupe Macroclimat et au groupe Edaphique-Topographique. En particulier, la Pluviométrie annuelle et la Profondeur du sol, retenues pour la stratification, sont indépendantes et efficaces, ce qui confirme leur valeur et contribue à valider l'étude. La salinité, exprimée par la conductivité, est une variable efficace mais très mal échantillonnée dans cette étude. Les variables décrivant les facteurs d'exploitation sont très peu efficaces.

\section{Les groupes écologiques}

Le tableau 1 présente les groupes écologiques qui résultent de l'étude, classés selon un gradient approximatif allant des " milieux humides" aux « milieux secs " ; pour chacun d'eux, outre la composition floristique, nous indiquons les caractéristiques écologiques essentielles, et les types biologiques (estimés) des espèces constitutives. Les taxons non pris en compte ont très souvent une fréquence trop faible pour être caractérisés. Dans un souci de simplification, nous avons volontairement omis les groupes qui n'apportent qu'une très faible information écologique (en particulier les groupes d'adventices, liés à la proximité aléatoire des cultures), ceux constitués d'espèces à très faible recouvrement, ainsi que certaines espèces plurimodales (Cynodon dactylon, Lippia nodiflora...).

Certains groupes importants, comme le Groupe à Paspalum-Kyllinga et, dans une moindre mesure, le groupe à Axonopus-Fimbristylis, présentent une compensation de facteurs entre l'« humidité climatique » (précipitations) et l'« humidité édaphique " (situation topographique) : présents dans tout le paysage dans les régions à pluviométrie supérieure à $1600 \mathrm{~mm}$, ils se réfugient peu à peu dans les bas-fonds humides en zone sèche. Le cas de figure inverse existe également.

\section{Les communautés végétales}

Le tableau 2 indique la composition en groupes écologiques des communautés mises en évidence. Ces communautés sont également ordonnées selon un gradient d'humidité; elles sont en outre regroupées en grands ensembles, en fonction du ou des groupes écologiques dominants. Il s'agit d'une ébauche de hiérarchisation, dans une perspective d'utilité pratique, et non pas dans le but de constituer une systématique des associations. Les numéros et lettres de référence sont arbitraires, et ne servent qu'à la correspondance avec le texte et les figures.

L'examen, même sommaire, du tableau 2 montre que les communautés correspondant à des conditions extrêmes (très humides ou très sèches) sont assez faciles à caractériser par leur composition en groupes écologi- 
TABLEAU 1

Groupes écologiques principaux des savanes de Grande-Terre, ordonnés selon un gradient d'humidité décroissante Main ecological species groups in the savannas of Grande-Terre, presented according to a decreasing humidity gradient

TB Type biologique (life form)

Cc Chamaephyte en coussinet (cushion-shaped chamaephyte)

$\mathrm{Ce} \quad$ Chamaephyte en espalier (mat-forming chamaephyte)

Cf Chamaephyte frutescent (frutescent chamaephyte)

$\mathrm{Cr} \quad$ Chamaephyte rampant (creeping chamaephyte)

Csf Chamaephyte suffrutescent (suffrutescent chamaephyte)

Gr Géophyte rhizomateux (rhizomatous geophyte)

HCc Hémicryptophyte cespiteux (tufted hemicryptophyte)

HCd Hémicryptophyte dressé (erect hemicryptophyte)
$\mathrm{HCg}$ Hémicryptophyte grimpant (climbing hemicryptophyte)

$\mathrm{HCr}$ Hémicryptophyte rampant (creeping hemicryptophyte)

HCrs Hémicryptophyte en rosette (rosulate hemicryptophyte)

HCs Hémicryptophyte à stolons (stoloniferous hemicryptophyte)

MP Macro-phanérophyte (macro-phanerophyte)

NP Nano-phanérophyte (dwarf phanerophyte)

$\mathrm{Pg} \quad$ Phanérophyte grimpant (climbing phanerophyte)

$T \quad$ Thérophyte (therophyte)

Les noms en CAPITALES sont ceux des espèces "principales" (cf. texte). Les espèces précédées d'une astérisque donnent leur nom au groupe. PRINTED names are those of the " main species" (see text). Species with an asterisk give the group their names.

\begin{tabular}{lcl}
\hline \hline \multicolumn{1}{c}{ COMPOSITION FLORISTIQUE } & T.B. & \multicolumn{1}{c}{ CARACTĖRES ÉCOLOGIQUES ESSENTIELS } \\
\hline $\begin{array}{l}\text { FIMBRISTYLIS CYMOSA R. Br. } \\
\text { PASPALUM VAGINATUM Sw. }\end{array}$ & $\begin{array}{c}\mathrm{HCc} \\
\mathrm{HCs}\end{array}$ & $\begin{array}{l}\text { Système lagunaire, falaises, littoral sableux. Forêt à Pterocarpus et forêt } \\
\text { xéro.-littor. Dépressions et plans bas. Pluv. < 1 400 mm. Halophile. }\end{array}$ \\
\hline * ECHINOCHLOA GUADELOUPENSIS & $\mathrm{Gr}$ & $\begin{array}{l}\text { Bordure interne de la forêt à Pterocarpus. Indifférent à la pluviométrie. } \\
\text { Situations humides sur sols hydrom. humifères acides de salinité variable. } \\
\text { (Hack.) Wegand }\end{array}$ \\
\hline Nitrophile.
\end{tabular}

ELEOCHARIS MUTATA (L.) R. \& S.

Gr qu'A. baccifera les évite.

* Cyperus alopecuroides Rottb.

* HYMENACHNE AMPLEXICAULIS (Rudge)

Gr Dépressions et plans inondables. Sols hydromorphes argileux divers.

Nees

POLYGONUM PUNCTATUM Ell.

$\mathrm{Gr}$

Csf

* BRACHIARIA MUTICA (Forsk.) Stapf

$\mathrm{HCs}$

Dépressions fermées et plans inondables, assez abrités du vent.

* PASPALUM DisTiCHUM L.

(P. paspalodes (Michx.) Scribn.)

HCs Dépressions et plans inondables. Sols hydromorphes divers. Indifférent à la pluviométrie dans le domaine étudié. Evite l'ombre.

* Neptunia plena (L.) Benth.

Paspalidium geminatum (Forssk.) Stapf

Paspalum pleostachyum Doell

$\mathrm{Cr}$ Dépressions et plans inondables. Sols divers sur argiles gonflantes, sables

* Sesbania sericea (Willd.) Link

$\mathrm{HCc} \quad$ calcaires, boues et vases, d'épaisseur variable. Zones peu pluvieuses.

Caperonia palustris (L.) St. Hil.

* Cyperus ochraceus Vahl

* Echinochloa colonum (L.) Link

$\mathrm{HCc}$

NP

* Eclipta alba (L.) Hassk.

T ? Parties basses et bas de pente, sur vertisols plus ou moins hydromorphes $\mathrm{HCc}$ ou sur régosols sur sable. Sols peu humifères. Groupe nitrophobe.

$\mathrm{HCc}$

Csf Dépressions et plans plus ou moins inondables. Sols hydromorphes divers e régosols sur sable. Préfère les stations abritées. Nitrophobe.

* Alternanthera sessilis (L.) R. Br.

$\mathrm{Cr}$ ? Sols hydromorphes divers, vertisols " complexes ", parfois régosols sur sable (creux très humides). $\mathrm{pH}<7,5$. Plaines de l'ouest et arrière-mangrove. Pluviométrie > $1400 \mathrm{~mm}$

* ERIOChloa POLYSTACHYA HBK. Mimosa pigra $\mathrm{L}$.

* PANICUM LAXUM Sw.

Pycreus polystachyos (Rottb.) Beauv.

Struchium sparganophorum (L.) O. Ktze.

Torulinium odoratum (L.) Hooper

Vigna luteola (Jacq.) Benth.

* Commelina diffusa Burm. f.

* Ludwigia octovalvis (Jacq.) Raven

* Desmodium adscendens (Sw.) DC.

Centrosema pubescens Benth.

Ipomoea setifera Poir.

* Kyllinga brevifolia Rottb.

* PASPALUM CONJUGATUM Berg.

Phyllanthus urinaria L.
$\mathrm{HCc} \quad$ Sols divers, plans, profonds, plus ou moins hydromorphes. $\mathrm{pH}<7$,5. Salinite

MP faible à moyenne. Pluviométrie $>1400 \mathrm{~mm}$. Plaines de l'ouest, arrière-

$\mathrm{HCc}$ mangrove, dépressions des Collines de Gosier.

$\mathrm{Gr}$

Csf

$\mathrm{HCc}$

$\mathrm{Pg}$

$\mathrm{Cr} \quad$ Groupe rudéral voisin du précédent, mais plus plastique : peut gagner des

Csf sols moins profonds et plus alcalins des pentes en zone très arrosée, et les basfonds en zone sèche. .

$\mathrm{Cr}$ Dépressions, plans et bas de pente sur sols plus ou moins hydromorphes, compacts à pâteux. $\mathrm{pH}<7,5$. Texture $\mathrm{AL}$ ou LA. Pluviométrie $>1600 \mathrm{~mm}$. Plaines de l'ouest, arrière-mangrove, parties basses des Grands-Fonds.

$\mathrm{Pg}$ Vertisols plus ou moins hydromorphes, vertisols " complexes ", sols hydro$\mathrm{Pg}$ morphes; sols vertiques en zone pluvieuse. Préfère les sols profonds et $\mathrm{Gr}$ argileux, mais peut gagner des sols moins épais en zone pluvieuse (comHCs pensation de facteurs). Pluviométrie $>1400 \mathrm{~mm}$ (surtout $>1600 \mathrm{~mm}$ ). T ? $\quad \mathrm{pH}<7,75$. Climax : surtout forêt mésophile et forêt à Pterocarpus. 
TABLEAU I (suite)

\begin{tabular}{|c|c|c|}
\hline COMPOSITION FLORISTIQUE & T.B. & CARACTĖRES ÉCOLOGIQUES ESSENTIELS \\
\hline $\begin{array}{l}\text { * AXONOPUS COMPRESSUS (Sw.) Beauv. } \\
\text { Desmodium incanum DC. var. } 1 \\
\text { Enicostemma verticillatum (L.) Engl. } \\
\text { * Fimbristylis dichotoma (L.) Vahl } \\
\text { Pseudelephantopus spicatus (Aubl.) Gleason } \\
\text { Psidium guajava L. }\end{array}$ & $\begin{array}{l}\mathrm{HCs} \\
\mathrm{Csf} \\
\mathrm{Csf} \\
\mathrm{HCc} \\
\mathrm{Csf} \\
\mathrm{MP}\end{array}$ & $\begin{array}{l}\text { Sols très divers, grumeleux à compacts. N'évite que les dépressions fermées } \\
\text { subaquatiques. Pluviométrie }>1400 \mathrm{~mm} \text {. Stations en moyenne moins humi- } \\
\text { des que le précédent, mais présente la même compensation de facteurs. } \\
\text { Climax : forêts xéro-mésophile, mésophile et à Pterocarpus. }\end{array}$ \\
\hline * Mimosa pudica L. & $\mathrm{Cf}$ & Pluviométrie $>1400 \mathrm{~mm}$, sur sols divers, de texture $\mathrm{A}$ ou $\mathrm{AL} . \mathrm{pH}<7,75$. \\
\hline * WEDELIA TRILOBATA (L.) Hitch. & $\mathrm{Cr}$ & $\begin{array}{l}\text { Rudérale peu indicatrice. Stations plus ou moins humides. Pluviométrie } \\
>1400 \mathrm{~mm} \text {. }\end{array}$ \\
\hline $\begin{array}{l}\text { * Digitaria cf. radicosa (Presl) Miq. } \\
\text { Elephantopus pilosus Philipson } \\
\text { Emilia sonchifolia (L.) DC. } \\
\text { Moghania strobilifera (L.) J. St.-Hil. } \\
\text { Solanum torvum Sw. }\end{array}$ & $\begin{array}{l}\mathrm{HCs} \\
\mathrm{HCrs} \\
\mathrm{T} \\
\mathrm{NP} \\
\mathrm{MP}\end{array}$ & $\begin{array}{l}\text { Rudérales des pentes fortes des Grands-Fonds et des buttes-témoins de la } \\
\text { Plaine des Abymes. Sols divers, sur calcaires ocres et socle de base, souvent } \\
\text { très pierreux. Pluviométrie }>1500 \mathrm{~mm} \text {. Préfère les stations assez ombragées. } \\
\text { Climax : forêts méso-xérophile et mésophile. }\end{array}$ \\
\hline $\begin{array}{l}\text { * STENOTAPHRUM SECUNDATUM (Walt.) } \\
\text { Ktze. }\end{array}$ & $\mathrm{HCs}$ & $\begin{array}{l}\text { Prairiale des pentes et sommets des Grands-Fonds et des buttes-témoins de la } \\
\text { Plaine des Abymes. Un écotype (?) en arrière-mangrove. Sols divers humifères } \\
\text { sur calcaires ocres. Struct. sablo-grum. à compacte. Nitrophile. }\end{array}$ \\
\hline
\end{tabular}

* Paspalum notatum Flugge

HCc Ecologie voisine de la précédente, mais mal caractérisée dans cette étude. Grands-Fonds et Collines de Gosier. Sols pentus et souvent pierreux.

* Mousses terrestres

* Pilea microphylla (L.) Liebm Anemia adiantifolia (L.) Sw.

Eupatorium odoratum L.

Eupatorium odoratum L.

* Haematoxylon campechianum L.

Lantana camara L.

Melochia nodiflora Sw.

* Randia aculeata L.
Sols calcaires tres courts et pierreux des fortes pentes des Cirands-Fonds et des $\mathrm{T}$ ? $\quad$ buttes-témoins de la Plaine des Abymes. $\mathrm{pH}>7,75$. Préfère les stations plus $\mathrm{HCc} \quad$ ou moins ombragées.

Asclepias curassavica L. Th

$\begin{array}{ll}\text { Asclepias curassavica L. } & T \\ \text { Cyperus sphacelatus Rottb. } & \text { HCc }\end{array}$

Indigofera spicata Forss.

NP Groupe subforestier des sommets, plans hauts et pentes fortes des collines MP (Grands-Fonds) et plateaux, sur rendzines, sols calc. mélan. et plus rarement NP fersiall. sur calcaires ocres ou blancs et tuf volcanique. Préfère les stations NP abritées. Forêts xérophile et xéro-mésophile.

PP

T Vertisols divers et sols vertiques, parfois rendzines sur sable ou tuf. Plaines et $\mathrm{HCc}$ plateaux. Pluviométrie $>1300 \mathrm{~mm}$. Sols grumeleux à compacts.

Alysicarpus vaginalis (L.) DC $\mathrm{Ce}$

* Blechum brownei Juss.

Ce Groupe principal de la prairie à Dichanthium quand la pluviométrie dépasse

Desmodium triflorum (L.) DC.

Csf

$\mathrm{Ce}$

$\begin{array}{ll}\text { Stapf } & \text { HCc }\end{array}$

Sporobolus cf. jacquemontii Kunth

$1200 \mathrm{~mm}$. N'évite que les bas-fonds très humides et les lithosols et régosols.

Teramnus labialis (L.f.) Spreng. $\quad \mathrm{HCg}$

Prairiale ubiquiste. Pluviométrie $>1000 \mathrm{~mm} . \mathrm{pH}>6,75$. N'évite que les parties basses inondables et les stations très sèches.

Fimbristylis (Abildgaardia) ovata (Burm. f.) HC

Kern

$\mathrm{HCc} \quad$ Prairiale ubiquiste très plastique. N'évite que les parties basses mal drainées. Evite l'ombre.

Brachiaria reptans (L.) Gard. \& C. E. Hubb. $\mathrm{HCc}$

Brachiaria subquadripara (Trin.) Hitch. HCs ?

* Desmanthus depressus H. \& B.

$\mathrm{Ce}$

* Ruellia tuberosa L.

Csf

* DICHANTHIUM ARISTATUM (Poir.)

$\mathrm{HCs}$

Prairiales et rudérales. Pluviométrie $<1500 \mathrm{~mm}$. N'évite que les régosols sur C. E. Hubb. sable et les sols hydromorphes. $\mathrm{pH}>7,75$. Forêts xérophile et xérophile littorale.

Kallstroemia pubescens (G. Don) Dandy

* STYLOSANTHES HAMATA (L.) Taubert

$\mathrm{HCr} \quad$ Lithosols, rendz. et sols calc. mélan. des plans hauts, sommets et pentes. Plu$\mathrm{Cr} \quad$ viométrie $<1400 \mathrm{~mm}$. pH 7,75 à 8,25 .

* Crotalaria verrucosa L.

Csf Plans et pentes faibles sur sols vertiques, fersiallitiques, ou rendzines. Pluvio-

* Indigofera tinctoria L. métrie $<1200 \mathrm{~mm}$. pH $>7,5$.

* BothrioChloa pertusa (L.) A. Camus HCs Brachiaria fasciculata (Sw.) S. T. Blake HCc Cenchrus echinatus L. Commelina elegans HBK. Croton flavens $\mathbf{L}$.

* Lantana involucrata $\mathbf{L}$.

Fond prairial de la savane à Dichanthium quand la pluviométrie est entre 950 et $1300 \mathrm{~mm}$. N'évite que les stations inondables, le sable et l'ombre forte.

* Pectis humifusa Sw. $\quad$ C

Sols pierreux assez peu épais : lithosols, rendz., sols calc. mélan., sols fersiall. sur récifs soulevés, sables littor., calc. blanc. Evite les sols argileux compacts. Pluviométrie $<1500 \mathrm{~mm}$. Plateaux et Collines du Gosier. Forêts xéro. et

$\mathrm{HCc}$

$\mathrm{Cr}$

NP

NP xéro.-littor.

Sols littoraux alcalins : lithosols, rendzines, sols fersiall. calcaires. Formations rases et piétinées. Préfère les vents forts et les embruns. 
TABLEAU 1 (suite)

\begin{tabular}{|c|c|c|}
\hline COMPOSITION FLORISTIQUE & T.B. & CARACTĖRES ÉCOLOGIQUES ESSENTIELS \\
\hline * Paspalum laxum Lam. & $\mathrm{HCc}$ & $\begin{array}{l}\text { Sols divers, pierreux et alcalins, peu profonds, peu argileux. Pluviométrie } \\
<1400 \mathrm{~mm} \text {. Forêts xéro. et xéro.-littor. Préfère les stations un peu ombra- } \\
\text { gées. }\end{array}$ \\
\hline $\begin{array}{l}\text { * Acacia tortuosa (L.) Willd. } \\
\text { Chloris inflata Link } \\
\text { Cucumis anguria L. } \\
\text { Euphorbia (Chamaesyce) prostrata Ait. } \\
\text { * Sida spinosa L. } \\
\text { Tragus berteronianus Schult. }\end{array}$ & $\begin{array}{l}\mathrm{MP} \\
\mathrm{HCc} \\
\mathrm{HCr} / \mathrm{T} \\
\mathrm{Ce} \\
\mathrm{Csf} \\
\mathrm{T} ?\end{array}$ & $\begin{array}{l}\text { Sols fersiall., lithosols, rendz., peu profonds et pierreux, peu argileux et peu } \\
\text { compacts, alcalins. Pluviométrie }<1400 \mathrm{~mm} \text {. Forêts xéro. et xéro.-littorale. }\end{array}$ \\
\hline $\begin{array}{l}\text { Acalypha chamaedrifolia (Lam.) Muell.-Arg. } \\
\text { Amaranthus crassipes Schlecht. } \\
\text { Bouteloua americana (L.) Scribn. } \\
\text { * DACTYLOCTENIUM AEGYPTIUM (L.) } \\
\text { Beauv. } \\
\text { * Portulaca oleracea L. } \\
\text { SIDA CILIARIS L. }\end{array}$ & $\begin{array}{l}\mathrm{Csf} \\
\mathrm{HCr} \\
\mathrm{HCc} \\
\mathrm{HCc} \\
\mathrm{T} ? \\
\mathrm{Csf}\end{array}$ & $\begin{array}{l}\text { Sols fersiall., lithosols, rendz., régosols sur sable, assez peu profonds, jamais } \\
\text { compacts, pierreux, très filtrants, sur pente faible ou nulle. Pluviométrie } \\
<1400 \mathrm{~mm} \text {. Forêts xéro. et xéro.-littor. Tolère le sel. }\end{array}$ \\
\hline $\begin{array}{l}\text { * Evolvulus convolvuloides (Willd.) Stearn } \\
\text { Heliotropium curassavicum L. } \\
\text { * SPOROBOLUS VIRGINICUS (L.) Kunth }\end{array}$ & $\begin{array}{l}\mathrm{Cr} \\
\mathrm{Cc} \\
\mathrm{HCs}\end{array}$ & $\begin{array}{l}\text { Sols fersiall., rendz. et sols calciques mélan. des bas-fonds des falaises, sur } \\
\text { récifs soulevés et calc. blanc. Préfère les stations très ensoleillées. Pluviomé- } \\
\text { trie }<1300 \mathrm{~mm} \text {. Halophile. Forêt xéro.-littor. }\end{array}$ \\
\hline $\begin{array}{l}\text { * Anthephora hermaphrodita (L.) Ktze. } \\
\text { * Digitaria bicornis (Lam.) R. \& S. var. ? } \\
\text { Lithophila muscoides Sw. sbsp. macrantha Urb. }\end{array}$ & $\begin{array}{l}\mathrm{T} ? \\
\mathrm{HCc} \\
\mathrm{Ce}\end{array}$ & Mêmes sols que le précédent. Pluviométrie $<1100 \mathrm{~mm}$. \\
\hline
\end{tabular}

ques. Pour les autres, c'est apparemment plus l'abondance que la simple présence d'un groupe qu'il faut prendre en compte.

Le tableau 3 présente les caractères floristiques et écologiques essentiels des communautés (dites « groupements »), dans le même ordre qu'au tableau 2 :

\section{Groupements à Dactyloctenium}

(1 à 5) Prairies littorales denses des zones sèches. Sols très souvent fersiallitiques, plus rarement rendzines, sols calciques mélanisés ou régosols sur sable. Climax : forêt xérophile littorale.

\section{Groupements à Dichanthium}

(8 à 16) Se rencontrent surtout sur les Plateaux, et sur les collines sèches des zones plus arrosées (compensation de facteurs). Les groupements 9 à 13 sont dominés par le Dichanthium aristatum et le Stylosanthes hamata, les autres (en conditions moins sèches) sont le domaine du groupe à Dichanthium annulatum et Blechum brownei. Climax : forêt xérophile le plus souvent.

\section{Groupements des Grands-Fonds}

$(17,18,20,21)$ Grands-Fonds et buttes-témoins de la Plaine des Abymes. Caractérisés par la présence plus ou moins abondante du Stenotaphrum secundatum, du Paspalum notatum et du groupe à Pilea et mousses. Pluviométrie en général supérieure à $1500 \mathrm{~mm}$. Climax : forêt xéro-mésophile. Le groupement 21 assure la transition avec les groupements suivants.

\section{Groupements à Axonopus et Paspalum}

$(23,24,25)$ Surtout dans les plaines de l'ouest et les fonds des régions de collines (plus rarement des plateaux). Les groupes à Axonopus-Fimbristylis et Paspalum-Kyllinga y sont dominants.

\section{Groupements très humides ou aquatiques des ré- gions sèches}

$(7,37,36,6)$ Parties basses humides des zones peu arrosées : bas-fonds, mares, marais et leurs abords, système lagunaire, littoral sableux inondable. Les groupements non aquatiques accueillent souvent des groupes écologiques des sols secs voisins.

\section{Groupements très humides ou aquatiques des régions pluvieuses}

$(26,27,28,30,31,32,33)$ Sols plats, profonds, argileux ou humifères, plus ou moins inondables ou inondés. La prise en compte du régime hydrique et salin annuel serait ici indispensable à une bonne compréhension de l'organisation de la végétation.

Dans ce tableau figurent également les valeurs pastorales moyennes. On observe de très grandes différences de valeur pastorale selon les communautés, depuis le groupement 1 (Pelouses littorales rases à Dactyloctenium aegyptium et Pectis humifusa des hauts de falaises sèches), sans aucun intérêt fourrager, jusqu'aux savanes denses et drues à Dichanthium aristatum sur vertisols en zone sèche du groupement 9 , qui peuvent atteindre les $3 / 4$ de la valeur d'une prairie à Pangola (Digitaria decumbens).

\section{E. L'analyse factorielle des correspondances}

\section{Analyse 350 Relevés $\times$ Abondances des 324 taxons (fig. 2)}

Les pourcentages d'inertie absorbée par les 6 premiers axes sont très faibles, et diminuent lentement (ce 
TABLEAU 2

Communautés végétales des savanes de Grande-Terre, ordonnées selon un gradient d'humidité croissante, et leur composition en groupes écologiques.

Plant communities in the savannas of Grande-Terre, presented according to a humidity gradient, and their composition in ecological species groups.

* Groupe très fréquent et très abondant (Group very frequent and very abundant).

$\mathrm{X}$ Groupe fréquent et/ou souvent abondant (Group frequent and/or often abundant).

$\mathrm{x}$ Groupe assez fréquent et/ou assez abondant (Group rather frequent and/or rather abundant).

: Groupe peu fréquent et jamais abondant (Group not frequent and never abundant).

\begin{tabular}{|c|c|c|c|c|c|c|c|c|c|}
\hline \multirow[b]{3}{*}{$\begin{array}{l}\text { Groupes } \\
\text { écologiques }\end{array}$} & \multicolumn{2}{|c|}{$\begin{array}{l}\text { Commu- } \\
\text { nautés } \\
\text { à } \\
\text { Dactylocten. }\end{array}$} & \multicolumn{2}{|c|}{$\begin{array}{l}\text { Communautés } \\
\text { à } \\
\text { Dichanthium }\end{array}$} & \multicolumn{2}{|c|}{$\begin{array}{l}\text { Comm. à } \\
\text { Stenotaph. } \\
\text { Gds. Fds. }\end{array}$} & \multirow{2}{*}{$\begin{array}{c}\text { C. à } \\
\begin{array}{c}\text { Axono. } \\
\text { et } \\
\text { Paspa. }\end{array} \\
\text { G }\end{array}$} & \multicolumn{2}{|c|}{$\begin{array}{l}\text { Communautés très humides } \\
\text { ou aquatiques } \\
\text { de zone } \\
\text { sèche }\end{array} \begin{array}{c}\text { de zone } \\
\text { pluvieuse }\end{array}$} \\
\hline & A & B & $\mathrm{C}$ & $\mathrm{D}$ & $\mathbf{E}$ & $\mathrm{F}$ & & $\mathrm{H}$ & I \\
\hline & $\begin{array}{ll}0 & 0 \\
1 & 2\end{array}$ & $\begin{array}{lll}0 & 0 & 0 \\
3 & 4 & 5\end{array}$ & $\begin{array}{lllll}0 & 1 & 1 & 1 & 1 \\
9 & 0 & 1 & 2 & 3\end{array}$ & $\begin{array}{llll}1 & 1 & 0 & 1 \\
4 & 6 & 8 & 5\end{array}$ & $\begin{array}{ll}1 & 1 \\
7 & 8\end{array}$ & $\begin{array}{ll}2 & 2 \\
0 & 1\end{array}$ & $\begin{array}{lll}2 & 2 & 2 \\
3 & 4 & 5\end{array}$ & $\begin{array}{llll}0 & 3 & 3 & 0 \\
7 & 7 & 6 & 6\end{array}$ & $\begin{array}{lllllll}2 & 2 & 3 & 2 & 3 & 3 & 3 \\
6 & 7 & 0 & 8 & 3 & 1 & 2\end{array}$ \\
\hline Paspalum vaginatum-Fimbristylis & & & & & & & & $*$ & $x$ \\
\hline $\begin{array}{l}\text { Echinochloa guadeloupensis } \\
\text { Bacopa-Ammania } \\
\text { Hymenachne-Cyperus } \\
\text { Brachiaria purpurascens }\end{array}$ & & & & & & & & $\begin{array}{l}\mathrm{x} x \\
\mathrm{x}\end{array}$ & $\begin{array}{cccc} & : & & * \\
\mathrm{X} X & * & * & \mathrm{x} \\
\mathrm{X} & * & : & \mathrm{X} \\
\mathrm{X} & : & \end{array}$ \\
\hline $\begin{array}{l}\text { Paspalum distichum } \\
\text { Neptunia-Sesbania } \\
\text { Echinochloa-Cyperus } \\
\text { Eclipta alba } \\
\text { Alternanthera sessilis }\end{array}$ & & $:$ & $:$ & & & & : & $\begin{array}{l}\mathrm{X} x \\
\mathrm{x} * \mathrm{X} \\
* \mathrm{X} x: \\
\quad:\end{array}$ & $\begin{array}{cccc}: & & & \\
& & : & : \\
\mathrm{X} & * & & \mathrm{x} \\
\mathrm{x} & \mathrm{x} & : & : \\
: & \mathrm{x} & \mathrm{x} & :\end{array}$ \\
\hline $\begin{array}{l}\text { Eriochloa-Panicum laxum } \\
\text { Ludwigia-Commelina diffusa } \\
\text { Desmodium adscendens } \\
\text { Paspalum conj.-Kyllinga } \\
\text { Axonopus comp.-Fimbristylis } \\
\text { Mimosa pudica } \\
\text { Wedelia trilobata }\end{array}$ & & & $\begin{array}{l}x \\
x \\
\vdots\end{array}$ & 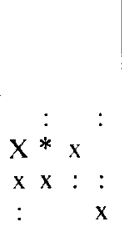 & $\begin{array}{cc}\vdots & \mathrm{x} \\
\mathrm{X} & * \\
\mathrm{x} & \mathrm{x} \\
\mathrm{x} & \mathrm{x}\end{array}$ & $\begin{array}{cc}: & \mathrm{x} \\
& \vdots \\
\mathrm{x} & \mathrm{X} \\
\mathrm{X} & * \\
: & \mathrm{X} \\
* & \mathrm{x}\end{array}$ & $\begin{array}{lll}: & & \mathrm{X} \\
\mathrm{x} & \mathrm{x} & * \\
\mathrm{x} & \mathrm{x} & \mathrm{x} \\
* & * & * \\
* & * & \mathrm{X} \\
* & \mathrm{x} & \mathrm{X} \\
\mathrm{x} & : & \mathrm{x}\end{array}$ & $\begin{array}{l}: \\
:\end{array}$ & 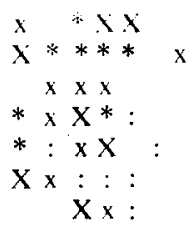 \\
\hline Elephant.-Digit. cf. radicosa & & & & & $: \mathrm{x}$ & $* \mathrm{x}$ & $* \mathrm{x}:$ & & : \\
\hline $\begin{array}{l}\text { Stenotaphrum secundatum } \\
\text { Paspalum notatum } \\
\text { Pilea micro.-Mousses }\end{array}$ & & & & & \begin{tabular}{cc|}
$*$ & $*$ \\
$\mathrm{x}$ & $\mathrm{x}$ \\
$:$ & $\mathrm{x}$
\end{tabular} & $\begin{array}{r}\vdots \\
\\
\\
X\end{array}$ & & & \\
\hline Haematoxylon-Randia & & & $: \mathrm{x} \quad \mathrm{X}$ & $: x \quad *$ & $* x$ & $\mathrm{X}$ : & $\mathrm{x}$ & & \\
\hline $\begin{array}{l}\text { Indigofera spicata } \\
\text { Dichanthium ann.-Blechum } \\
\text { Teramnus labialis } \\
\text { Abildgaardia ovata }\end{array}$ & & $\begin{array}{l}: \\
:\end{array}$ & 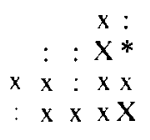 & $\begin{array}{l}: \mathrm{X} \\
* * * * \\
\mathrm{x}: \\
\mathrm{XX} \times \mathrm{x}\end{array}$ & $\begin{array}{ll}* & \mathrm{x} \\
\mathrm{x} & \mathrm{x}\end{array}$ & $\begin{array}{l}\mathrm{x} \\
\mathrm{X}^{*} \\
\mathrm{x}\end{array}$ & $\begin{array}{ccc} & x & : \\
X & X & X \\
: & : \\
: & x & \end{array}$ & $\begin{array}{l}\mathrm{x} \\
\mathrm{x} \\
:\end{array}$ & $\begin{array}{lll}x & & \\
* & x & x \\
x & x \\
x & \end{array}$ \\
\hline $\begin{array}{l}\text { Ruellia tuberosa-Desmanth. } \\
\text { Dichanthium aristatum } \\
\text { Stylosanthes hamata } \\
\text { Indigofera-Crotalaria verrucosa } \\
\text { Bothriochloa-Lantana involucrata } \\
\text { Pectis humifusa } \\
\text { Paspalum laxum } \\
\text { Acacia tort.-Sida spinosa }\end{array}$ & $\begin{array}{r}\mathrm{x} \\
\mathrm{X} \\
\mathrm{X} \\
\mathrm{X}\end{array}$ & $\begin{array}{ccc}\mathrm{X} & \mathrm{X} & \mathrm{x} \\
& \vdots & \\
* & * & * \\
& \vdots & : \\
\mathrm{x} & \mathrm{X} & : \\
\vdots & \mathrm{X} & \\
\mathrm{X} & \mathrm{X} & *\end{array}$ & 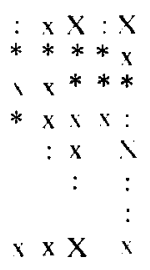 & $\begin{array}{l}\mathrm{X} \times \mathrm{X}: \\
: \\
: \quad: \\
: \quad \mathrm{x}\end{array}$ & $\mathrm{x}$ & : & & $\begin{array}{c}\mathrm{X} \\
:\end{array}$ & $x$ \\
\hline $\begin{array}{l}\text { Dactyloctenium-Portulaca } \\
\text { Evolvulus-Sporobolus virg. } \\
\text { Anthephora-Digitaria }\end{array}$ & $\begin{array}{ll}* & * \\
* & X\end{array}$ & $\begin{array}{ccc}* & * & * \\
: & : & \mathrm{x} \\
\mathrm{x} & \mathrm{x} & \mathrm{x}\end{array}$ & & & & & & : & \\
\hline
\end{tabular}

qui est habituel pour ce type de données) : 1 : 3,61 p. $100,2: 3,38$ p. $100,3: 2,88$ p. 100 , $4: 2,77$ p. $100,5: 2,57$ p. $100,6: 2,20$ p. 100 . On remarque l'existence d'un effet Guttman très marqué, qui semble indiquer qu'un seul grand facteur synthétique suffit à expliquer la répartition constatée (BACHACOU, 1973) ; ce facteur synthétique est sans doute approché de très près par la variable « Humidité appa- rente » (fig. 2b), qui, de toute évidence, intègre en particulier la Pluviométrie (pour sa branche droite) et la Fréquence de submersion (pour sa branche gauche).

Sur la figure 2a sont portées, outre les groupements, les principales espèces. Les groupes écologiques ne sont pas représentés. Cette analyse ne permet de caractériser que les groupements correspondant aux milieux très humides ou très secs: les autres (ou relevés 
TABLEAU 3

Communautés végétales des savanes de Grande-Terre : physionomie et principaux caractères écologiques. VP : Valeur pastorale moyenne. Plant communities in the savannas of Grande-Terre : physionomy and main ecological features. VP: Mean pasture value.

$\mathrm{N}^{\circ} \quad$ Caractères physionomiques et floristiques essentiels
Caractères écologiques

essentiels
V.P.

1 Prairies littorales très rases à Dactyloctenium, Sporobolus virginicus et Pectis humifusa.

2 Très voisin du précédent, avec apparition du Groupe à RuelliaDesmanthus et du Groupe à Bothriochloa-Lantana.

3 Prairies littor. très rases. Graminées cespiteuses dominantes. Voisin des précédents, avec les groupes à Anthephora-Digitaria, Bothriochloa-Lantana et Stylosanthes.

4 Voisin du précédent. Faible recouvrement des herbacées. Abondance des groupes rudéraux (non évoqués ici).

5 Prairies littor. épaisses à fort recouvrement. Apparition de quelques groupes écologiques des sols lourds et humides.

9 Prairies épaisses et denses à Dichanthium aristatum, Indigofera-Crotalaria, Stylosanthes et Teramnus. Présence épisodique du groupe à Neptunia-Sesbania.

10 Prairies assez épaisses, voisines des précédentes, sans groupe indicateur des sols humides. Abildgaardia et groupe à Ruellia fréquents. Abondance des postculturales.

11 Prairies rases, à Stylosanthes et Dichanthium aristatum. Groupes à Bothriochloa-Lantana, Acacia-Sida et Haematoxylon fréquents et abondants. Teramnus rare.

12 Prairies à Dichanthium aristatum et Stylosanthes hamata; le le groupe à Dichanthium annulatum est assez fréquent, ainsi qu'Indigofera spicata.

13 Prairies très communes, denses mais d'épaisseur variable, à Dichanthium annulatum, Stylosanthes hamata, Abildgaardia. Abondance des groupes de rudérales et d'arvales non pris en compte ici. Le gr. à Bothriochloa-Lantana est fréquent, ainsi que le groupe subforestier à Haematoxylon.

14 Prairies denses d'épaisseur variable; groupe à Dichanthium annulatum très largement dominant. Groupe à Axonopus abondant et fréquent, ainsi qu'Abildgaardia et les groupes postculturaux non indiqués ici. Mimosa pudica fréquent.

16 Prairies assez rases mais denses, à Dichanthium annulatum et Axonopus, assez voisines des précédentes, mais avec Indigofera spicata et le groupe subforestier à Haematoxylon.

8 Prairies épaisses à Dichanthium annulatum et Axonopus.

15 Prairies épaisses et denses, mal caractérisées, à Dichanthium annulatum, Haematoxylon, Wedelia, et groupes postculturaux (non pris en compte ici)

17 Groupement à Stenotaphrum secundatum et à DichanthiumBlechum. Groupe à Axonopus-Fimbristylis fréquent mais peu abondant. Groupe subforestier à Haematoxylon fréquent et abondant.

18 Groupement à Stenotaphrum secundatum et à AxonopusFimbristylis. Le groupe à Dichanthium-Blechum devient secondaire. Apparition du groupe à Paspalum-Kyllinga.

20 Groupement à Wedelia trilobata. Abondance du groupe rudéral à Elephantopus-Digitaria et du groupe rupicoìe à Pileamousses.

21 Groupement à Axonopus-Fimbristylis et Dichantium-Blechum. Le groupe à Paspalum-Kyllinga est souvent présent.

Hauts de falaises littorales ensoleillées. Sols courts, alcalins, 10 salés : fersiallitiques ou calciques mélanisés.

Situation plus sèche. Sols plus courts, moins salés (rendzines et 11 calc. mélan.). Affleurements rocheux fréquents.

Rochers, falaises et sables littoraux. Sols assez superficiels, 41 de salinité variable, souvent faible. S. fersiall., rendz., régosols/sable. Affleurements rocheux fréquents.

Parties plates ou peu pentues des rochers et falaises littoraux, 33 sur sols fersiall. et rendzines de faible salinité.

Sols plats, lourds, profonds, des hauts de falaises littor, 44 sèches : sols fersiallitiques et calciques mélanisés.

Sols vertiques et vertisols (souvent plus ou moins hydromor- 74 phes) plans et épais, très argileux, non pierre!ıx, compacts, de $\mathrm{pH}$ voisin de 7 . Pluv. $<1200 \mathrm{~mm}$. Plateau est.

Sols divers, plus secs et plus pierreux que les précédents, 66 grumeleux à compacts, souvent en bas de versant. Pluviométrie $<1200 \mathrm{~mm}$ (jusqu'à $1400 \mathrm{~mm}$ ). Plateaux est et moyen.

Rendzines et sols calc. mélan. assez superficiels, de haut de 54 versant, sablo-grumeleux à grumeleux-compacts, souvent pierreux. Pluv. comme le précédent. Plateaux est et moyen.

Rendzines, sols calciques mélanisés, sols fersiall., vertisols 68 parfois à tendance hydromorphe. Milieux et bas de versants. Pluviométrie 1 100-1 $400 \mathrm{~mm}$. Plateaux est et moyen.

Sols calc. mélan., rendzines diverses, plus rarement sols ver- 43 tiques ou vertisols ; gravier souvent abondant. Pentes fortes des hauts de versants, et sommets. Pluv. $1100-1500 \mathrm{~mm}$ (jusqu'à $1700 \mathrm{~mm}$, sur sol squelettique). Plateaux, collines de Gosier, sommets très secs des Grands-Fonds.

Sols cal. mélan., vertiques, vertisols, en situation plus humide que le précédent (par la pluviométrie ou la topographie), peu pierreux. Plateaux, collines de Gosier. Parfois sur sols très filtrants en zone très arrosée (compensation).

Vertisols souvent plus ou moins hydromorphes, sols vertiques 50 et calc. mélan., compacts, plans, profonds, Pluv. 1300 $1700 \mathrm{~mm}$. Plateaux, bas de pente de Grands-Fonds.

Vertisols plus ou moins hydromorphes, bas et plans, inon- 44 dables par fortes pluies. Pluviométrie $1000-1400 \mathrm{~mm}$. Plateaux (bas-fonds) et arrière-mangrove.

Sols calciques mélanisés de hauts de versants sous pluviométrie 52 moyenne.

Pentes fortes et sommets des Grands-Fonds et des buttestémoins de la Plaine des Abymes, sur sols calc. mélan., rendzines, sols vertiques peu épais, pierreux. Calcaires ocres. Sols en général bien pourvus en azote. Pluv. $>1500 \mathrm{~mm}$.

Conditions voisines du précédent, mais un peu plus humides : pente moins forte et/ou sol plus épais et plus argileux ; ombrage souvent assez marqué. Plans hauts et bas de pente. Sols comme dans le précédent, et vertisols " complexes"

Rendzines et sols calc. mélan. très peu épais et très pierreux. 41 Pentes fortes, dégradées par l'érosion consécutive au déboisement des sommets des Grands-Fonds.

Dépressions, plans bas, bas de pente, dans les mêmes lieux que les trois groupements précédents, sur vertisols souvent hydromorphes et vertisols " complexes ». Pluv. $>1400 \mathrm{~mm}$. 
TABLEAU 3 (suite)

\begin{tabular}{ll}
\hline No & \multicolumn{1}{c}{$\begin{array}{c}\text { Caractères physionomiques et floristiques } \\
\text { essentiels }\end{array}$} \\
\hline 23 & $\begin{array}{l}\text { Prairies très denses, à Paspalum, Axonopus, Mimosa pudica, } \\
\text { avec une grande abondance des groupes à Sida-Hyptis (rudéral, } \\
\text { non pris en compte ici) et à Elephantopus-Digitaria. Appari- } \\
\text { tion du Desmodium adscendens. }\end{array}$
\end{tabular}

24 Prairies très denses, souvent rases, essentiellement à Paspalum et Axonopus.

25 Prairies plus épaisses que les précédentes, à Paspalum-Kyllinga et à Ludwigia-Commelina ; le groupe à Eriochloa-Panicum Paricum apparaît, traduisant bien l'humidité du milieu.

7 Groupement à Echninochloa-Cyperus et à Lippia (non pris en compte ici). Présence de nombreux groupes prairiaux et rudéraux des sols secs voisins. Prairies épaisses et denses.

37 Prairies subaquatiques peu denses, mais hautes, à NeptuniaSesbania et Paspalum distichum.

36 Prairies aquatiques plus ou moins denses, à NeptuniaSesbania et Ipomoea aquatica (non pris en compte ici). Le groupe à Bacopa-Ammania apparaît parfois.

6 Prairies le plus souvent littorales, à Paspalum vaginatum Fimbristylis cymosa et à Lippia Modiflora. Des groupes des sols plus secs voisins interviennent parfois.

26 Prairies à Dichanthium annulatum, Axonopus et Paspalum, avec présence fréquente des groupes à Ludwigia-Commelina, Eriochloa-Panicum, Echinochloa-Cyperus et Lippia nodiflora.

27 Prairies très épaisses à Ludwigia-Commelina et à EchinochloaCyperus ; les groupes à Dichanthium, à Pasp.-Kyllinga et à Axonopus sont ici presque absents. Plusieurs groupes caractérisent les sols humides (Hymenachne-Cyp. surtout).

30 Prairies à Eriochloa-Panicum et Ludwigia-Commelina. Le Wedelia trilobata, et les groupes à Paspalum-Kyllinga et à Bacopa-Ammania sont souvent présents.

28 Prairies à Bacopa-Ammania, Ludwigia-Commelina et Paspalum conjugatum-Kyllinga. Les groupes à Axonopus, à Eriochloa-Panicum et le Lippia nodiflora sont relativement fréquents. Epaisseur du tapis herbacé très variable.

33 Prairies souvent épaisses, à Hymenachne-Cyperus, EriochloaPanicum laxum et Ludwigia-Commelina.

31 Groupement modal à Bacopa-Ammania. Formations en géné ral très denses mais très rases.

32 Groupements à Echinochloa guadeloupensis : formations très épaisses (plus d'un mètre) et très denses.

Vertisols « complexes ", vertisols plus ou moins hydromorphes et sols vertiques, parfois calc. mélan. Grands-Fonds et Plaine des Abymes. Dépressions, plans bas, bas de pente. Pluv. > $1500 \mathrm{~mm}$. Voisin du groupement 21, en situation un peu plus pluvieuse.

Sols ferallitiques, vertisols « complexes » et vertisols plus ou moins hydromorphes, très profonds et plans, très peu humifères, souvent acides. Climax : forêt mésophile.

Vertisols « complexes » et vertisols plus ou moins hydromorphes, plans, très profonds, acides, mal drainés. Plaine de Grippon, et bas-fonds : Grands-Fonds et collines de Gosier.

Surtout sur vertisols hydromorphes, plans, inondables par fortes pluies, de profondeur, texture et compacité variées. Pluviométrie $<1500 \mathrm{~mm}$.

Vertisols hydromorphes. Système lagunaire, et bas-fonds des plateaux. Pluviométrie $<1200 \mathrm{~mm}$ surtout.

Pluviométrie $<1200(-1300) \mathrm{mm}$. Vertisols hydromorphes 42 sur argiles gonflantes. Drainage interne nul : sols inondés presque toute l'année (mares, marais). Salinité variable.

Groupement halophile littoral. Pluv. en général $<1500 \mathrm{~mm}$. Sols hydromorphes, vertisols hydrom., et régosols sur sables salés. Syst. lagunaire et littoral sableux inondable.

Vertisols plus ou moins hydrom., vertisols « complexes » principalement, parfois sols calc. mélan. plans sous forte pluviométrie. Plans bas et dépressions des plateaux et de la plaine de Grippon. Salinité souvent non négligeable. Pluv. $>1200 \mathrm{~mm}$. Forme transition avec les gr. 23, 24 et 25 .

Vertisols hydromorphes et sols hydromorphes plans, très profonds, peu humifères. Dépressions et plans bas des plateaux et de la plaine de Grippon. Pluv. $>1400 \mathrm{~mm}$. Salinité faible ou nulle.

Sols hydromorphes divers, plans, très profonds, en zone d'arrière-mangrove très pluvieuse. Salinité souvent non négligeable. $\mathrm{pH}<7,5$. Pluv. $>1600 \mathrm{~mm}$.

Conditions de milieu très voisines de celles du groupement précédent, mais salinité en moyenne nettement plus forte. Arrière-mangrove.

Pluv, assez variable, $>1200 \mathrm{~mm}$. Sols hydromorphes divers, de salinité variable, souvent faible, formés sur argiles diverses. Submersion, fréquente à continue. Arrière-mangrove, mares en bas-fond, ravines.

Conditions de milieu comme dans les groupements 28 et 30,27 mais sols nettement et constamment salés et toujours pâteux. Arrière-mangrove : lieux dégagés et ensoleillés.

Conditions de milieu comme dans les groupements 28,30 et 31: ombrage en général assez fort, du fait de la proximité immédiate de la forêt à Pterocarpus. Sols hydromorphes de salinité variable, sur boues et vases.

« moyens »), les plus nombreux, sont en effet condensés en un noyau central difficile à analyser. C'est pourquoi une étude de ces seuls relevés moyens a été entreprise.

\section{Analyse 260 Relevés « moyens » $\times$ Importance des groupes dans les relevés}

Les pourcentages d'inertie absorbée par les 6 pre- miers axes sont les suivants : $1: 10,12$ p. $100,2: 6,16$ p. $100,3: 5,21$ p. $100,4: 5,01$ p. $100,5: 4,63$ p. 100 , $6: 4,39$ p. 100 . Les figures 3,4 et 5 présentent les cartes factorielles (plan 1-2) relatives à cette analyse ; elles sont à rapprocher des tableaux 1 à 3 , dont elles constituent un bon résumé en ce qui concerne les groupes et groupements «moyens».

La discrimination des communautés, plus facile du fait de l'élimination des relevés " extrêmes », est de 

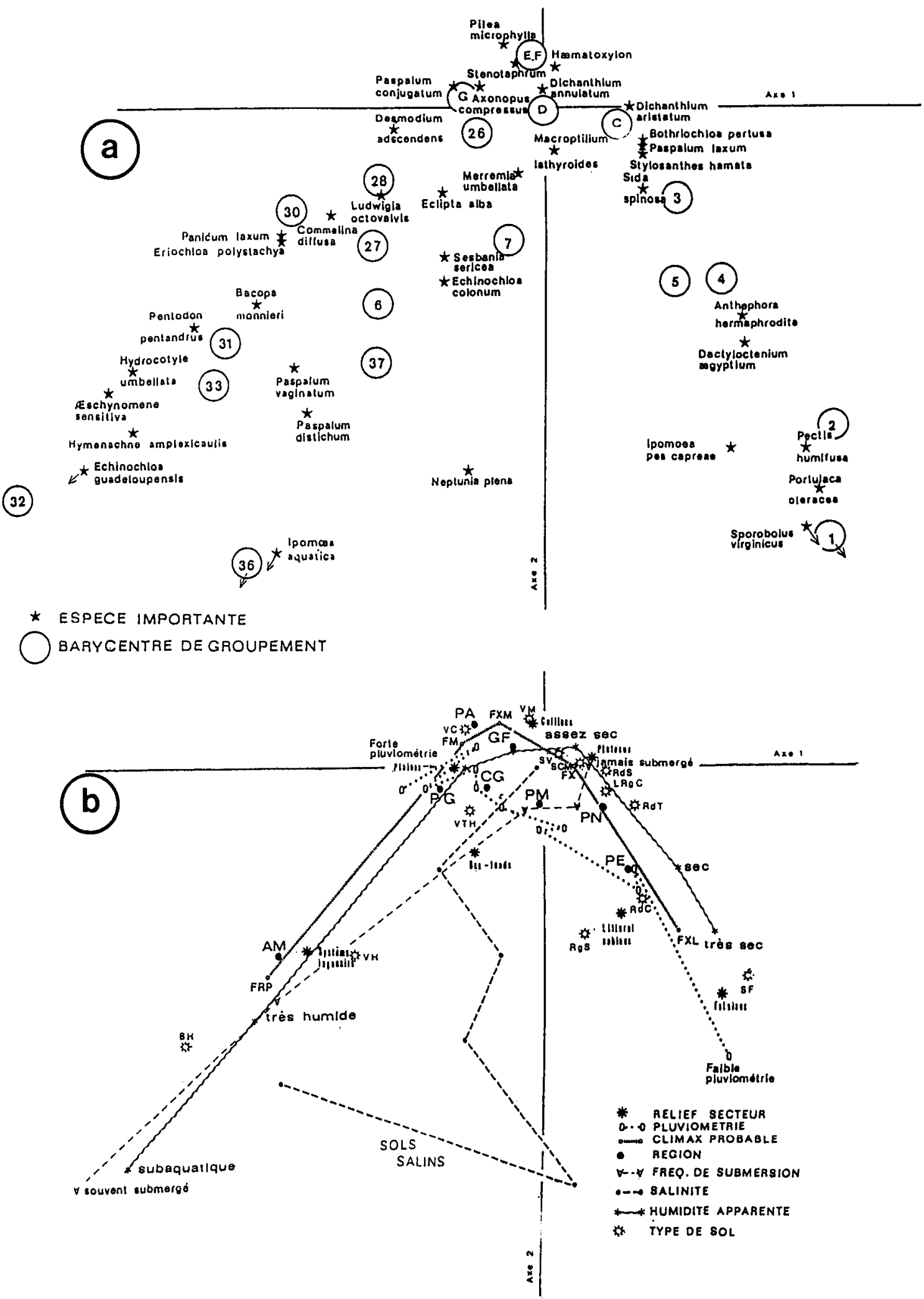

Figure 2

Projection dans le plan 1-2 de l'AFC Relevés $\times$ Abondances.

a) Communautés végétales, et espèces importantes.

b) Quelques variables du milieu.

Projection on 1-2 plane of the correspondence factor analysis (AFC) for samples $\times$ species abundances.

a) Plants communities, and important species.

b) Some ecological factors.

Climax : FXL : Forêt xérophile littorale (littoral xerophilous forest), $F X$ : Forêt xérophile (xerophilous forest), FXM: Forêt xéromésophile (xero mesophilous forest), FM : Forêt mésophile (mesophilous forest), FRP : Forêt rivulaire à Pterocarpus (riparian Pterocarpus forest).
Région (cf. fig. 1) : PE : Plateau Est, PM : Plateau moyen, PN : Plateau Nord, GF: Grands-Fonds, $C G$ : Collines de Gosier, PA : Plaine des Abymes, PG : Plaine de Grippon, AM : Mangrove et arrière-mangrove.

Type de sol: SF: Sol fersiallitique (Red calcic cambisol), $R g S$ : Régosol sur sable calcaire (Arenosol on calcareous sand), RdC: Rendzine sur calcaire (Rendzina on limestone), RdT: Rendzine sur tuf (Rendzina on powdery limestone), RdS: Rendzine sur sable (Rendzina on calcareous sand), $L \mathrm{RgC}:$ Lithosol ou régosol sur calcaire (Lithosol on limestone), SCM : Sol calcique mélanisé (Calcic phaeozem), $S V$ : Sol vertique (Vertic cambisol), VM: Vertisol modal (Chromic vertisol), VC: "Vertisol complexe " ou sol ferrallitique (Gleyic vertic cambisol, or ferralic cambisol), VTH : Vertisol à tendance hydromorphe (Gleyic vertisol), $V H:$ Vertisol hydromorphe (Vertic gleysol), SH : Sol hydromorphe (Eutric gleysol). 
Figure 3

Projection des relevés dans le plan l-2 de l'AFC 260 Relevés $\times$ Groupes écologiques (les symboles se réfèrent au tableau 2).

Projection of samples on 1-2 plane of AFC 260 Samples $\times$ Groups (symbols refer to table 2).
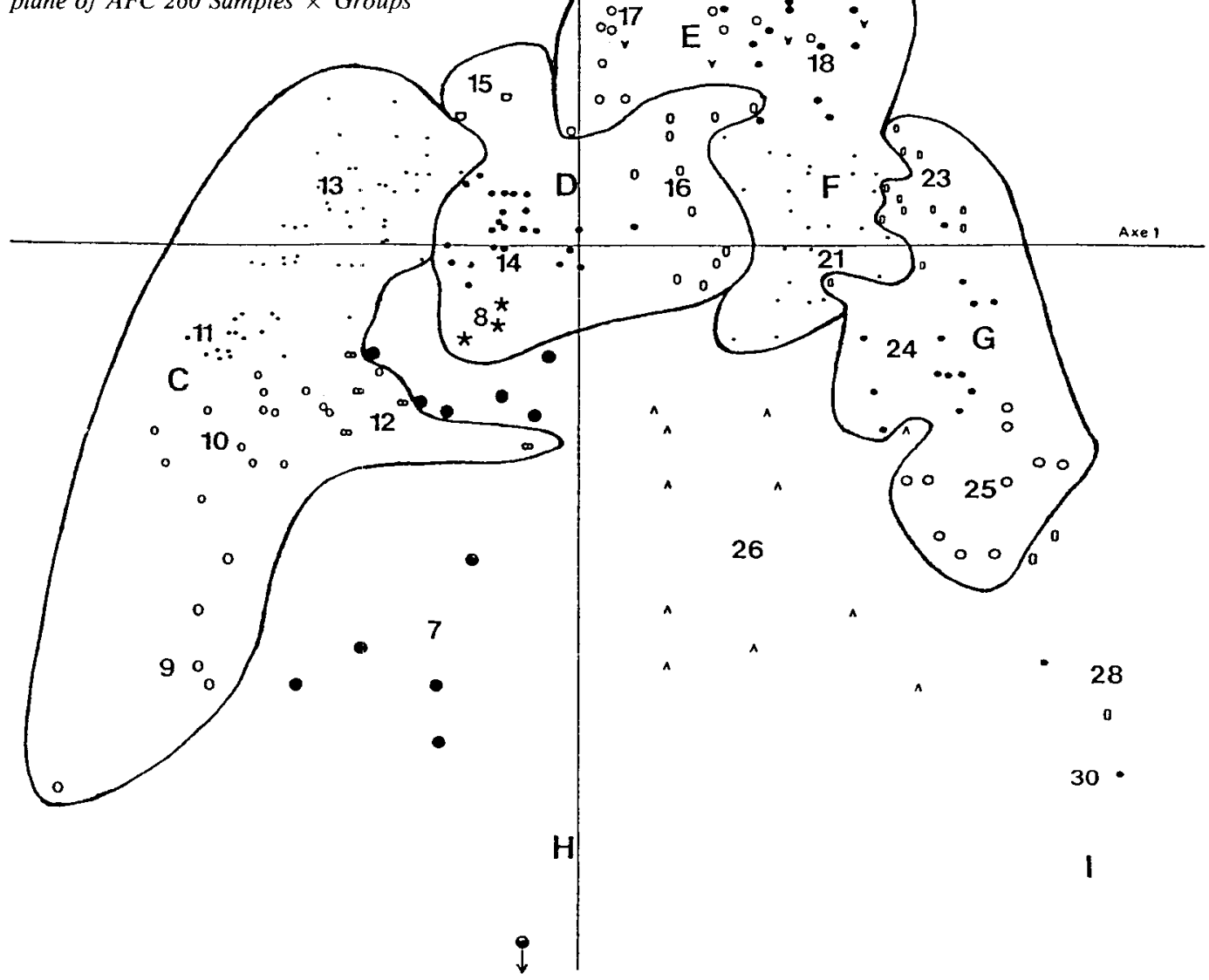

Figure 4

Projection des groupes écologiques dans le plan 1-2 de l'AFC 260 Relevés $\times$ Groupes écologiques.

Projection of ecological species groups on 1-2 plane of AFC 260 Samples $\times$ Groups.

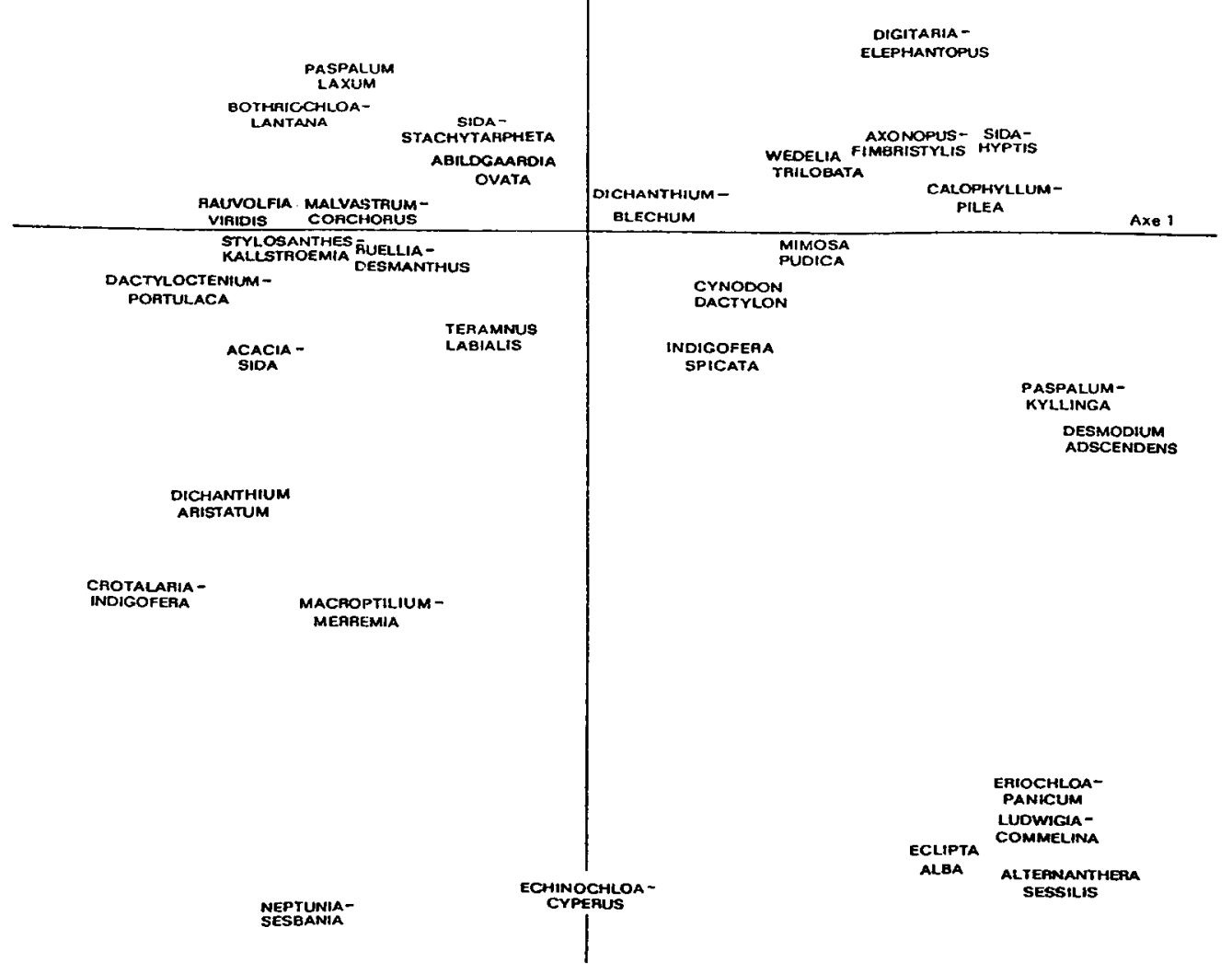



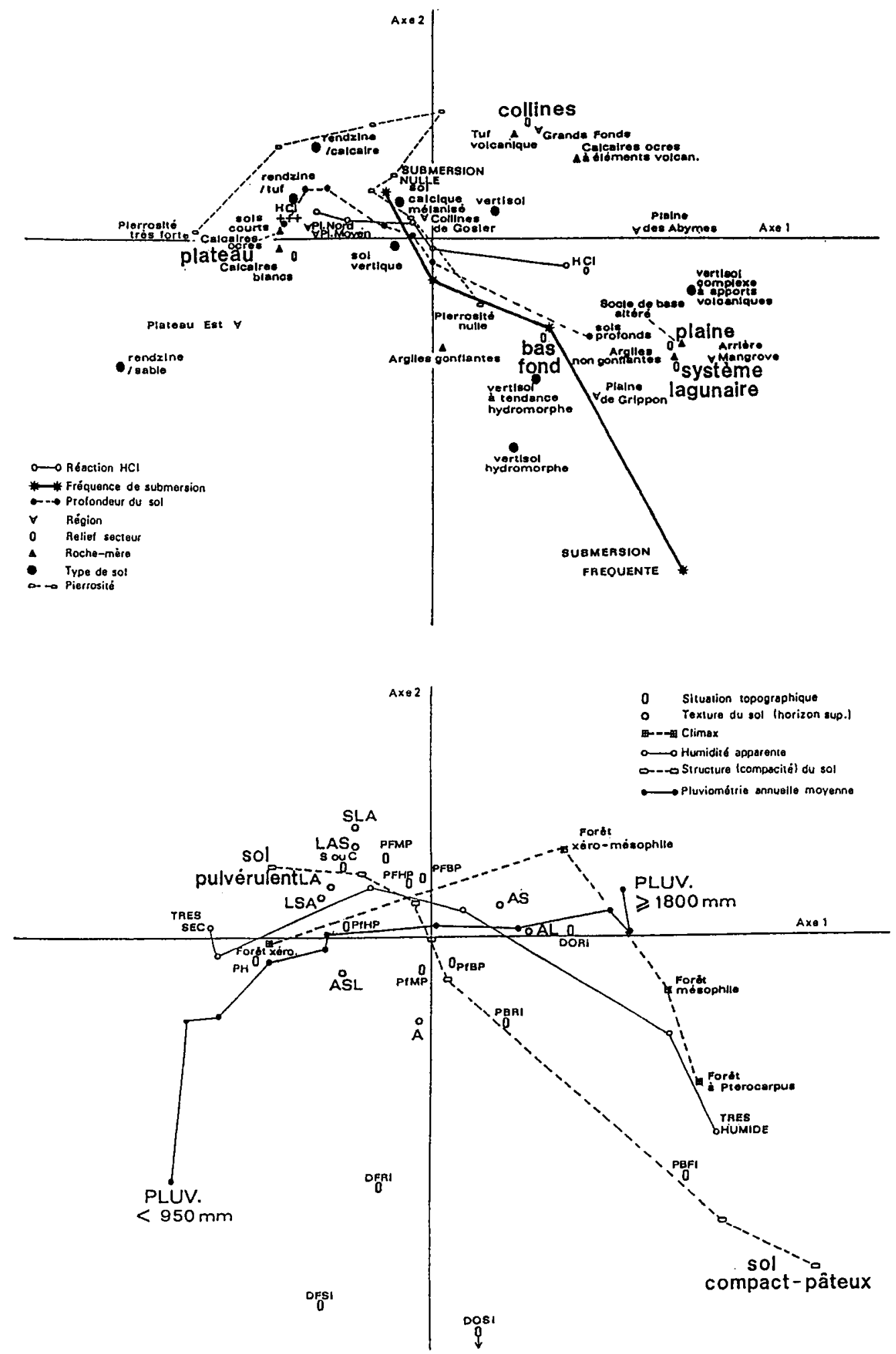

Figure 5

Projection de quelques variables (en "variables supplémentaires ») dans le plan 1-2 de l'AFC 260 Relevés $\times$ Groupes écologiques.

Situation topographique:

DF : Dépression fermée.

DO : Dépression ouverte.

$P B \quad:$ Plan bas.

$P H \quad$ : Plan haut.

$\mathrm{Fl} \quad$ : Facilement inondable.

RI : Rarement inondé

$B P \quad$ : Bas de pente.

$M P \quad$ : Mi-pente.

$S$ ou $C:$ Sommet ou crête.

$S I \quad$ : Souvent inondé.

$P F \quad:$ Pente faible.

$P F \quad:$ Pente forte.

HP : Haut de pente.
Projection of some ecological factors (as subsidiary items) on 1-2 plane of AFC 260 Samples $\times$ Groups.

Topographic situation :

$D F \quad:$ closed depression.

$D O \quad$ : open depression.

$P B \quad$ : low plane.

$P H \quad$ : high plane.

FI : easily flooded.

$R I \quad:$ seldom flooded.

$B P \quad$ : lower part of slope.

$M P \quad$ : half-way uphill.

$S$ ou C: Top or crest.

$S I \quad$ : often flooded.

$P F \quad$ : faint slope.

$P F \quad:$ steep slope.

$H P \quad:$ upper part of slope 
plus fortement accentuée par la prise en compte de l'« importance » des groupes écologiques dans les relevés. L'effet Guttman est ici beaucoup moins marqué, ce qui tend à montrer l'existence de plusieurs facteurs relativement indépendants. On remarque en effet, par exemple, que l'axe 1 est expliqué principalement par la Pluviométrie et le Climax probable (variables très liées), et l'axe 2 par la Fréquence de submersion, la Compacité du sol, la Situation topographique, la Pierrosité (qui sont également liées entre elles).

\section{F. Les « paysages prairiaux » des petites régions}

Le tableau 4 présente un synoptique des groupements par petite région.

\section{Les Plateaux calcaires}

Les 3 plateaux (régions 1,2 et 3 ) peuvent être décrits ensemble. Les savanes, sauf sur sol très humide, y subissent un arrêt complet de la végétation à la saison sèche.

\section{a) Les pelouses littorales}

Les pelouses littorales sur calcaire (le plus souvent sur les hauts de falaises) sont en général composées de l'un des groupements à Dactyloctenium. Leur valeur pastorale est faible, surtout pour les groupements 1 et 2 (sur sols peu épais et très salés), composés de groupes écologiques pratiquement sans aucune espèce fourragère. Les groupements 3,4 et 5 sont un peu meilleurs, grâce à la présence des groupes à Stylosanthes hamata et à Anthephora-Digitaria.

b) Les savanes à Petit Foin des sols moyens ou secs, non submersibles

Ce sont de loin les plus importantes des plateaux calcaires. Physionomiquement, elles donnent une impression de continuum, en raison de l'abondance toujours très forte de l'une ou de l'autre des 2 espèces de Dichanthium (groupements 8 à 16, qui concernent 123 relevés sur 350 ). Leur valeur pastorale moyenne varie entre 40 et 75 , ce qui indique leur intérêt. Ces bonnes valeurs sont dues en grande partie aux Dichanthium, mais également au Teramnus labialis et au Stylosanthes hamata.

c) Les savanes des bas-fonds et des sols humides ou aquatiques

- Sols plus ou moins salés

Il s'agit le plus souvent du système lagunaire (creux en arrière d'un cordon littoral, par exemple), ou parfois d'une partie inondable du littoral sableux. C'est le groupement littoral à Paspalum vaginatum et Fimbristylis cymosa qui domine. Sa valeur pastorale est très mauvaise, et les possibilités d'amélioration en sont à peu près nulles.

- Sols non ou très peu salés

Sols inondables seulement par fortes pluies :

On rencontre ici essentiellement le groupement 7 apparenté au groupement 8 des bas de pente - caractérisé par l'abondance du groupe à Echinochloa-
Cyperus. La valeur pastorale est assez bonne, et le fait que la situation topographique élimine pratiquement l'effet des périodes sèches est un encouragement aux essais d'amélioration. Cependant, ces sols souvent fertiles seraient sans doute mieux employés à la culture fourragère qu'à la savane, même améliorée.

Sols souvent inondés, stations subaquatiques ou aquatiques :

On y trouve essentiellement les groupements 37 (prairies subaquatiques) et 36 (prairies aquatiques). Ces groupements se rencontrent dans les bas-fonds les plus humides des plateaux, sur les bords de mares, ainsi que dans les marais littoraux, où ils assurent très souvent la transition avec les prairies de l'arrièremangrove. Ils sont caractérisés par les groupes à Neptunia-Sesbania et à Paspalum distichum, ce dernier conduisant, avec le Neptunia, à une valeur pastorale non négligeable. Cependant les possibilités d'amélioration sont faibles si le drainage est impossible.

\section{Les Grands-Fonds}

Sur leur bordure orientale, les Grands-Fonds se raccordent progressivement au Plateau de Saint-François. On y trouve des savanes très semblables. Plus à l'ouest, l'individualité des savanes des Grands-Fonds s'affirme, sous la double influence de la pluviométrie croissante et des roches-mères différentes.

Sur les pentes, on rencontre très fréquemment le groupement 18, caractérisé par la dominance du Stenotaphrum secundatum. Ces savanes ont une assez bonne valeur pastorale.

Sur les hauts de pentes et les sommets, en situation plus sèche, c'est le groupement 17 qui prédomine, du moins si les affleurements rocheux ne sont pas trop abondants. Le Dichanthium annulatum est ici aussi abondant que le Stenotaphrum, et la valeur pastorale est bonne. Ces savanes, souvent situées à proximité des bosquets relictes des sommets, ont tendance à être colonisées par le groupe subforestier à HaematoxylonRandia, ce qu'un bon entretien devrait éviter. Sur les pentes les plus fortes, où le sol est très peu épais, et les affleurements rocheux fréquents, on rencontre le groupement 20, qui comporte essentiellement le Wedelia trilobata et le groupe rupicole à Pilea et Mousses. Sa valeur pastorale est faible.

Les bas de pentes sont le plus souvent recouverts par le groupement 21, à Axonopus, Paspalum conjugatum et Dichanthium annulatum, qui passe progressivement, dans les bas-fonds, au groupement 23, à Paspalum conjugatum, Axonopus et Mimosa pudica, assurant la transition avec la Plaine des Abymes. La valeur pastorale est très moyenne.

\section{Les Collines de Gosier}

Cette région rappelle la bordure orientale des Grands-Fonds et le Plateau de Saint-François. Cependant, localement, on peut retrouver certains groupements typiques des Grands-Fonds voisins, en particulier le groupement 17, à Stenotaphrum et Dichanthium annulatum. La "Grande Ravine " qui sépare cette région des Grands-Fonds est floristiquement proche de la zone de transition entre la Plaine de Grippon et l'Arrière-mangrove (voir ci-après). 
TABLEAU 4

Importance relative des communautés végétales herbacées dans les petites régions de Grande-Terre, par référence à une répartition aléatoire. Relative importance of herbaceous plant communities in the subregions of Grande-Terre (compared to random distribution).

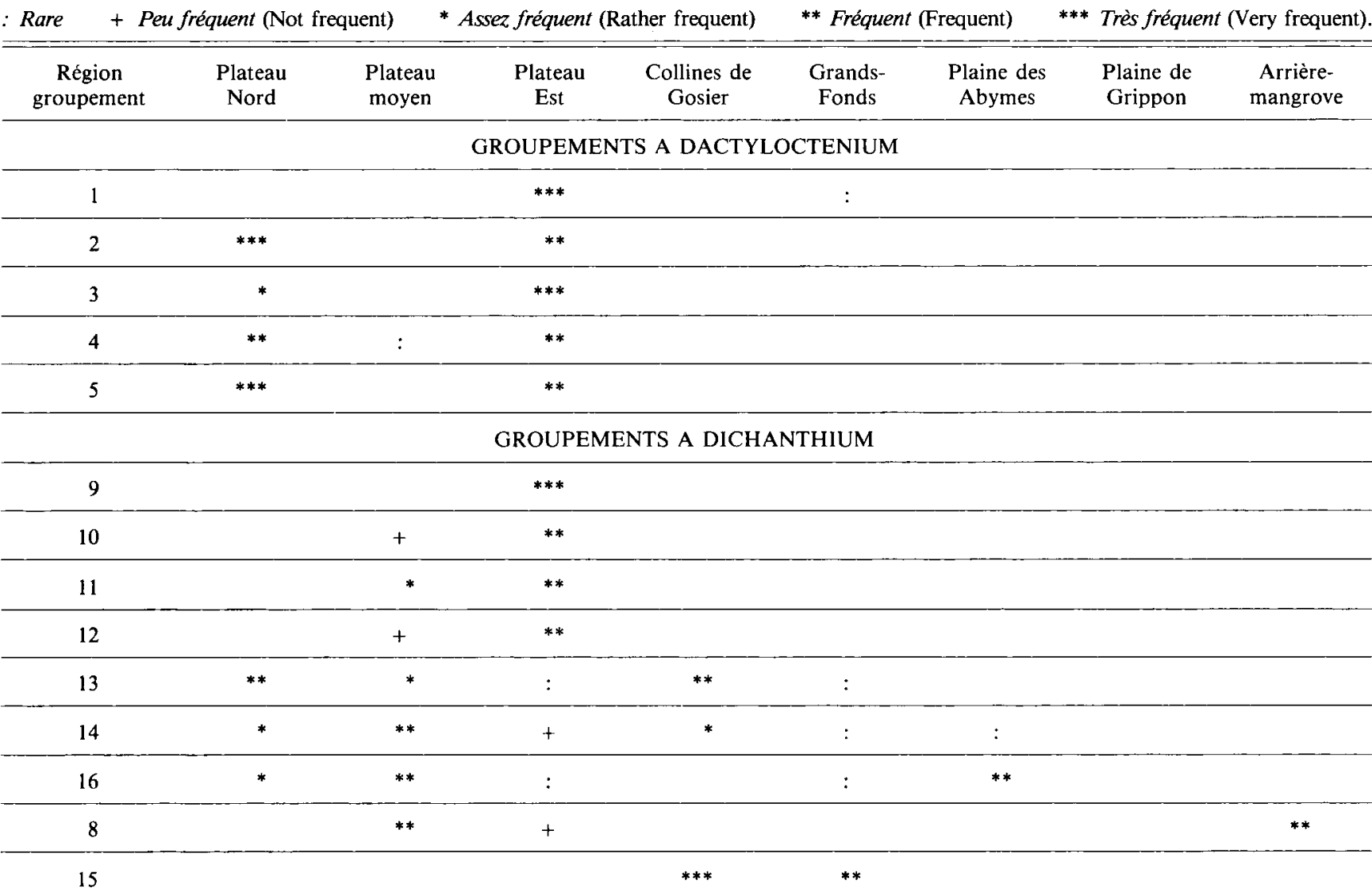

\section{GROUPEMENTS A STENOTAPHRUM}

17

18

20

21

GROUPEMENTS A AXONOPUS ET PASPALUM

23

24

25

\begin{tabular}{lllll} 
& $* * *$ & $* * *$ & & \\
\hline$* *$ & $:$ & $* * *$ & $* *$ & + \\
\hline & $:$ & + & $* * *$ & $* * *$ \\
\hline
\end{tabular}

GROUPEMENTS DES SOLS HUMIDES OU AQUATIQUES

7

37

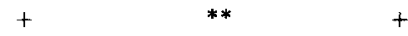

36

*

$6+2 * *$

$26 \quad * *+$

27

30

33

28 


\section{Les Plaines de l'ouest}

\section{a) Caractères communs}

La plupart des savanes des zones très plates (qui prédominent dans la Plaine de Grippon) sont constituées par le groupement 24 , comportant presque exclusivement les groupes à Axonopus-Fimbristylis et à Paspalum-Kyllinga. Dans les stations mal drainées, on rencontre le groupement 25 , très voisin, mais comportant des groupes des sols humides: LudwigiaCommelina, et Eriochloa-Panicum. La valeur pastorale est assez médiocre, du fait de l'abondance des " herbes sûres » peu appétantes (Axonopus compressus et Paspalum conjugatum). Les bas de pente sont souvent occupés, comme dans les Grands-Fonds, par le groupement 21 , plus riche en Dichanthium annulatum.

\section{b) Particularités de la Plaine de Grippon}

Dans sa transition avec l'arrière-mangrove du Grand Cul-de-Sac Marin se rencontrent quelques groupements de sols humides à subaquatiques : le groupement 26, médiocre, voisin du groupement 25 , le groupement 33, où domine le Groupe à HymenachneCyperus et surtout le groupement 27 , meilleur grâce à Brachiaria mutica, Eriochloa polystachya, Commelina diffusa, Echinochloa colonum.

\section{c) Particularités de la Plaine des Abymes}

Les nombreuses buttes-témoins se rapprochent beaucoup des Grands-Fonds : on y trouve les mêmes groupements 17 et 18 .

\section{L'« Arrière-mangrove »}

Les communautés végétales sont ici davantage liées aux conditions d'humidité et de salinité du sol qu'au climat (communautés " azonales »). Une description de l'organisation de la végétation de l'arrièremangrove suppose une étude approfondie des régimes de l'eau et du sel dans le sol, ainsi que la connaissance de l'histoire des parcelles; cela sort du cadre de l'étude, beaucoup de groupements de cette région étant impropres au pâturage.

On retrouve ici plusieurs groupements des sols humides déjà rencontrés dans d'autres régions. En revanche, 4 groupements assez répandus sont typiques de l'arrière-mangrove : le groupement 32, à Echinochloa guadeloupensis, rarement pâturé. Les groupements 28 et 31 , largement dominés par le groupe à Bacopa monnieri, petite Scrophulariacée rase sans intérêt fourrager. Le groupement 30, à Eriochloa-Panicum et à Ludwigia-Commelina, surtout en zone très pluvieuse.

\section{DISCUSSION ET CONCLUSION}

Plusieurs grands types de « savanes » ont été mis en évidence, et caractérisés par leur composition floristique en relation avec le milieu. Les grands traits de la répartition des espèces sont expliqués par le macroclimat et par les caractères topographiques et pédologiques.

Les variables liées au mode d'exploitation sont notées de façon insuffisamment précise pour jouer un rôle important. Il en est difficilement autrement dans une étude générale portant sur un territoire relativement étendu. Il conviendrait d'affiner les résultats par des études limitées à quelques-uns des groupements ici caractérisés ; ces études, dans lesquelles l'abondance des espèces devrait être prise davantage en compte, permettraient en particulier d'avoir une idée des déplacements d'équilibre floristique induits par telle ou telle pratique d'exploitation.

Cependant nos résultats, même s'ils manquent de précision sur certains points, sont utilisables dans une optique d'amélioration des prairies naturelles de Grande-Terre. Sans empiéter sur le domaine des agronomes et des zootechniciens, nous pensons utile de faire figurer ici quelques éléments de réflexion au sujet des essais d'enrichissement en légumineuses.

- Il est nécessaire, pour chaque espèce de légumineuse essayée, de réaliser des essais dans chacun des groupements végétaux importants mis ici en évidence. C'est l'une des raisons d'être de la présente étude.

- Il faudra élargir l'éventail des espèces de légumineuses essayées, sans oublier les espèces locales intéressantes, qui ont le mérite d'être adaptées : le Teramnus labialis, le Vigna luteola et le Stylosanthes hamata peuvent raisonnablement être pris en compte dans un programme d'enrichissement.

Les efforts d'amélioration doivent porter en priorité sur les prairies que nous avons appelées « moyennes», qui sont les plus utilisées par l'élevage :

- Bien que leur production s'annule en saison sèche, les prairies à Dichanthium annulatum ou D. aristatum, abondantes sur les plateaux, sont relativement bonnes. Au D. aristatum est le plus souvent associé le Stylosanthes hamata, particulièrement abondant sur les sols peu épais en zone sèche. Dans les zones plus arrosées, le $D$. annulatum est très souvent associé au Teramnus labialis. Dans les 2 cas, il faut chercher en priorité à maintenir ou à favoriser des associations GraminéesLégumineuses déjà existantes et potentiellement intéressantes. Il est important de noter que l'abondance du Teramnus semble très liée aux conditions d'exploitation et à l'histoire de la parcelle ; cela permet d'espérer qu'il sera possible d'accroître cette abondance.

- Les prairies à Axonopus-Paspalum des plaines de l'ouest sont généralement sous-évaluées - y compris dans la présente étude - du fait de l'appétence assez faible de l'Axonopus compressus et du Paspalum conjugatum, comparée à celle des Dichanthium. Cependant, la valeur nutritive de ces 2 espèces est meilleure que celle des Dichanthium (XANDÉ, 1985), et les animaux les ingèrent en quantités importantes lorsqu'ils n'ont pas le choix; de plus, ces savanes souffrent moins de la sécheresse que celles à Dichanthium. Sauf sur sol humide, où le Vigna luteola est présent et devrait être favorisé, on n'y trouve guère de légumineuse intéressante. Aucun essai d'enrichissement n'a encore été effectué dans ces communautés ; les résultats obtenus sur les savanes à Dichanthium ne sont certainement pas applicables ici.

- Il est difficile d'envisager l'amélioration des prairies à Stenotaphrum des Grands-Fonds, en raison des fortes pentes ; elles comprennent également assez peu de légumineuses intéressantes, et leur enrichissement 
par des techniques simples et peu onéreuses (semis à la volée sans préparation, par exemple), devrait être étudié. L'éventail des espèces envisageables est ici très ouvert, et le Teramnus (fréquent mais peu abondant) y a sa place.

Enfin, dans les milieux à forte contrainte, aucun investissement important n'est envisageable, sauf cas particulier. Cependant, certains essais mériteraient d'être conduits :

- Sur les meilleures pelouses littorales sèches (groupements $3,4,5$ ), il conviendrait d'étudier l'enrichissement éventuel en Stylosanthes hamata, ou en un autre Stylosanthes xérophile (SOMDA, 1981).

- Dans les prairies humides des bas-fonds des plateaux, des plaines de l'ouest et de l'« arrièremangrove ", l'étude de l'enrichissement en Vigna luteola - ou en toute autre espèce supportant l'excès d'eau temporaire dans le sol - mériterait d'être abordée.

Il serait hasardeux, en l'état actuel des connaissan- ces, d'extrapoler sans précaution les résultats de la présente étude à d'autres îles de la Caraïbe, même à celles qui présentent des conditions analogues de climat et de sols. En effet, le cloisonnement lié à l'insularité induit des variations non négligeables dans la fréquence relative des espèces, dans leur patrimoine génétique, et donc très certainement dans leur comportement écologique. Cependant, nous pensons que certaines conclusions, moyennant quelques études locales complémentaires, peuvent se révéler utiles hors de la Guadeloupe.

Reçu le 8 janvier 1987. Accepté le 30 juillet 1987.

\section{REMERCIEMENTS}

A Y.-M. CABIDOCHE, de la Station Agropédoclimatique (I.N.R.A., Antilles-Guyane), qui a bien voulu assurer la détermination des sols, et à $O$. SoBESKY, qui en a effectué l'analyse physicochimique, nous adressons nos très chaleureux remerciements.

\section{RÉFÉRENCES BIBLIOGRAPHIQUES}

Bachacou J., 1973. L'effet Guttman dans l'analyse de données phytosociologiques. I.N.R.A., Département de Biométrie et de Calcul automatique. Document 73/5, $30 \mathrm{p}$.

Daget Ph., Godron M., 1982. Analyse fréquentielle de l'écologie des espèces dans les communautés. Masson, Paris, $163 \mathrm{p}$.

Daget Ph., Poissonnet J., 1971. Une méthode d'analyse phytologique des prairies. Ann. agron., $22: 1,5-41$.

De Crécy J., 1969. Les vertisols sur calcaire aux Antilles. Problème d'utilisation agricole. Caribbean Food Crops Society, VII Congrès annuel, Fort-de-France. Vol. VII, 251-265.

De Wet J. M. J., Harlan J. R., 1968. Taxonomy of Dichanthium section Dichanthium (Gramineae). Bol. Soc. Argent. Bot., 12, 206227.

Doreau M., Vivier M., 1979a. La prairie naturelle à Dichanthium caricosum en Guadeloupe. I. Effet de la saison sur la production, la composition chimique et la digestibilité in vitro. Agronomie tropicale, XXXIV, 4, 356-361.

Doreau M., Vivier M., 1979b. La prairie naturelle à Dichanthium caricosum en Guadeloupe. II. Composition botanique, composition chimique des principales espèces et préférences alimentaires des bovins. Agronomie tropicale, XXXIV : 4, 362-371.

Fournet J., 1978. Flore illustrée des Phanérogames de Guadeloupe et de Martinique. I.N.R.A., Paris, 1654 p.

Fournet J., 1983. Approche phytoécologique de l'amélioration fourragère des jachères dans un transect de la péninsule sud d'Haïti. Acta aecolog., OEcol. applic., 4 : 3, 273-296.

Garcia-Molinari O., 1952. Grasslands and grasses of Puerto Rico. Bull. 102, Univ. Puerto Rico, Agric. Experiment Station, Rio Piedras, Puerto Rico.

Godron M., 1971. Essai sur une approche probabiliste de l'écologie des végétaux. Th. Doct. d'Et. Univ. Sc. Techn. du Languedoc, $247 \mathrm{p}$.

Gounot M., 1969. Méthodes d'étude quantitative de la végétation. Masson, Paris, 314 p.

Gruet P., 1984. Quelques aspects de l'homogénéité floristique des prairies à Dichanthium de Grande-Terre (Guadeloupe). Mémoire de fin d'étude ISTOM, $43 \mathrm{p}$.

Guillerm J. L., 1971. Calcul de l'information fournie par un profil écologique et valeur indicatrice des espèces. OEcol. plant., 6, 209225 .

Henrard J. Th., 1950. Monograph of the genus Digitaria. Universitaire Pers Leiden. Leiden, 999 p.
Howard R. A., 1977. Flora of the lesser Antilles. II. Pteridophyta (by G. R. Proctor). Arnold Arboretum, Harvard University, 414 p. Howard R. A., 1979. Flora of the lesser Antilles. III. Monocotyledonae. Arnold Arboretum, Harvard University, $586 \mathrm{p}$.

Lasserre G., 1961. La Guadeloupe. Etude géographique. Union Française d'Impression, Bordeaux, $1135 \mathrm{p}$.

Meredith D., 1955. The grasses and pastures of South Africa. Central News Agency. $771 \mathrm{p}$.

Oakes A. J., Butcher J. O., 1962. Poisonous and injurious plants of the US Virgin Islands. Agric. Res. Service, USDA, Miscell. Public. $\mathrm{n}^{\circ} 882,97 \mathrm{p}$.

Roberty G., 1960. Monographie systématique des Andropogonées du globe. Boissiera $9,455 \mathrm{p}$.

Somda J.-Ph., 1981. Variabilité et morphogenèse chez différents taxons du genre Stylosanthes (Légumineuse Papilionacée) en liaison avec leur exploitation agronomique et leurs possibilités d'amélioration comme plantes fourragères de fauche. Thèse Univ. Paris-Sud, $233 \mathrm{p}$.

Stehlé H., 1935. Flore de la Guadeloupe et des Dépendances. I. Essai d'Ecologie et de Géographie botanique. Imprimerie Catholique, Basse-Terre, $284 \mathrm{p}$.

Stehlé H., 1937a. Les associations végétales de la Guadeloupe et leur intérêt dans la valorisation rationnelle. Rev. de Bot. tropic. appl. et d'Agron. iropic., 186 et 187.

Stehlé H., 1937b. Esquisse des associations végétales de la Martinique. Fort-de-France.

Stehlé H., 1950. Pasture and fodder grass development and improvement in the French Caribbean territories. Carib. Comm., Port-ofSpain, Trinidad.

Stehlé H., 1951. Les pâtures naturelles et les cultures fourragères dans l'Archipel Caraïbe. Ann. Amél. Plantes, 1, 548-559.

Stehlé H., 1956. Survey of Forage Crops in the Caribbean. Carib. Comm., Port-of-Spain, Trinidad, 389 p.

Vélez I., 1957. Herbaceous angiosperms of the Lesser Antilles. Ed. Biology Dept. Inter-american Univ., Puerto-Rico. 121 p.

Xandé A., Garcia-Trujillo R., Caceres O., 1985. Tableaux de la valeur alimentaire des fourrages tropicaux de la zone caraibe. I.N.R.A.-I.C.A., 51 p.

Xandé A., 1985. La productivité des pâturages : problèmes posés et intérêt de quelques techniques pour améliorer la productivité dans le cadre des petites exploitations. Coll. " Systèmes de production agricole caribéens et alternatives de développement ». Martinique, 911 mai 1985. 